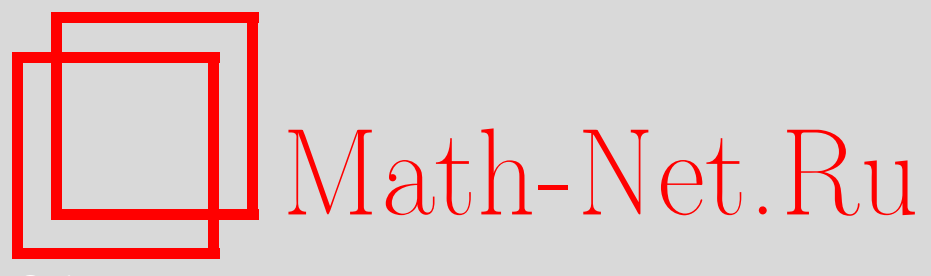

П. Г. Гриневич, С. П. Новиков, Сингулярные конечнозонные операторы и индефинитные метрики, $У \mathrm{MH}$, 2009, том 64, выпуск 4, 45-72

DOI: https://doi.org/10.4213/rm9307

Использование Общероссийского математического портала Math-Net.Ru подразумевает, что вы прочитали и согласны с пользовательским соглашением http://www . mathnet.ru/rus/agreement

Параметры загрузки:

IP : 34.239 .49 .27

26 апреля 2023 г., 12:52:09

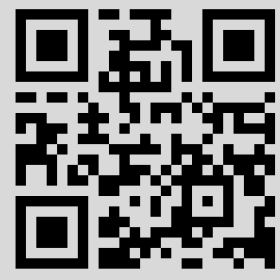




\section{Сингулярные конечнозонные операторы и индефинитные метрики}

\section{П. Г. Гриневич, С. П. Новиков}

Во многих задачах "вещественные" спектральные данные для конечнозонных периодических операторов (состоящие из римановой поверхности с отмеченной "бесконечно удаленной точкой", локального параметра в этой точке и дивизора полюсов) порождают операторы с вещественными сингулярными коэффициентами. Эти операторы не являются самосопряженными в обычном гильбертовом пространстве функций переменной $x$ (с положительной метрикой). В частности, эта ситуация имеет место для операторов Ламе с эллиптическим потенциалом $n(n+1) \wp(x)$, волновые функции которых были найдены Эрмитом в XIX веке. Однако, в соответствии с идеями работ [1]-[4], именно такие функции Бейкера-Ахиезера служат правильными аналогами дискретных и непрерывных базисов Фурье на римановых поверхностях. Оказывается, что для рода $g>0$ эти операторы симметричны относительно неположительно определенного (индефинитного) скалярного произведения, описанного в данной работе. Аналог непрерывного преобразования Фурье оказывается изометрией в этой метрике. Мы также описываем образ этого преобразования Фурье в пространстве функций переменной $x \in \mathbb{R}$.

Библиография: 24 названия.

Ключевые слова: спектральная теория, сингулярные конечнозонные операторы, индефинитные гильбертовы пространства, непрерывные базисы Фурье-Лорана на римановых поверхностях, потенциалы Ламе, модель Калоджеро-Мозера.

\section{СОДЕРЖАНИЕ}

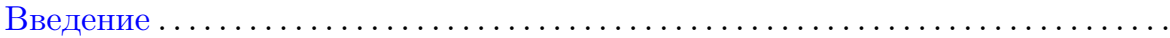

1. Канонические контуры и скалярные произведения функций Бейкера-

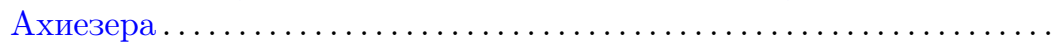

2. Индефинитные скалярные произведения на гиперэллиптических римановых поверхностях. Операторы Шрёдингера с сингулярными потенциалами . . . . . . . . . . . . . . . . . . . . . . . . . .

Работа выполнена при поддержке РФФИ (грант № 08-01-00054-а), программы "Ведущие научные школы" (грант НШ-1824.2008.1), а также программы Президента РАН "Фундаментальные проблемы нелинейной динамики".

(C) П. Г. Гриневич, С. П. Новиков, 2009 
2.1. Описание индефинитного пространства $\widetilde{H}_{L}$ для вещественных сингулярных конечнозонных $x$-периодических операторов $L$ об-

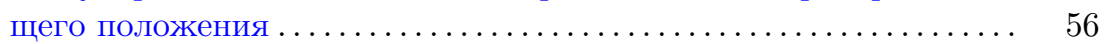

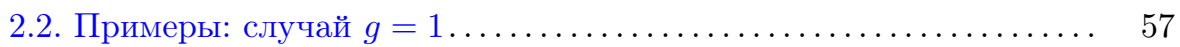

Приложение А. Ядро Коши-Бейкера-Ахиезера . . . . . . . . . . . . . . 60

Приложение В. Гиперэллиптический случай. Периодические граничные

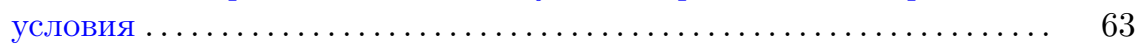

В.1. Ядро Коши-Бейкера-Ахиезера ....................... 63

В.2. Задача с периодическими граничными условиями ........... 64

В.3. Обобщения: промежуточные и однополюсные конечнозонные сингулярные потенциалы . . . . . . . . . . . . . . . . . . 68

B.4. Построение бесконечнозонных потенциалов с конечным $r$,

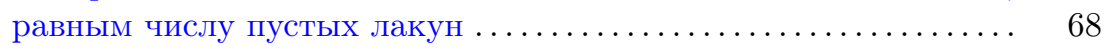

В.5. Потенциалы с одним полюсом ...................... 69

В.6. Примеры: вырожденные случаи ...................... 69

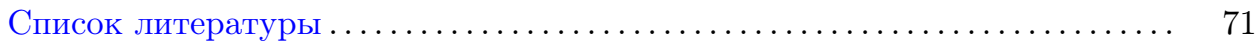

\section{Введение}

После того, как в 1974 г. была проинтегрирована конечнозонными методами периодическая задача для знаменитого уравнения КдФ, были построены обширные семейства так называемых функций "Бейкера-Ахиезера" (БА) на римановых поверхностях.

Они были использованы для решения периодической задачи для уравнений КдФ, КП и ряда других уравнений теории солитонов таких, как нелинейное уравнение Шрёдингера, синус-Гордон. Спектральная теория одномерного и двумерного операторов Шрёдингера развивалась, начиная с 1974 г., с использованием анализа на римановых поверхностях. В 1987 г. было открыто, что специальные функции Бейкера-Ахиезера порождают аналоги базисов Лорана-Фурье для функций и тензорных полей на римановых поверхностях (базисы и алгебры Кричевера-Новикова были построены в [1]-[3]). Эти базисы были использованы для многопетлевого (отвечающего роду $g>0$ ) операторного квантования замкнутой бозонной струны. Другие идеи, близкие к гармоническому анализу со спектральным параметром на римановых поверхностях и оказавшиеся полезными для нас, были развиты в работе [5].

Авторами данной работы в приложении к [4] было отмечено, что эти конструкции позволяют построить аналог непрерывного преобразования Фурье. Данная работа является прямым продолжением [4]. В значительной степени она мотивирована следующей задачей.

ЗАДАчА. Рассмотрим одномерный оператор Ламе $L=-\partial_{x}^{2}+u(x)$ с потенциалом $u(x)=n(n+1) \wp(x)$, где $\wp(x)$ - эллиптическая функция Вейерштрасса с полюсами на вещественной оси (см. [6]). Существует ли разумная спектральная теория для таких операторов на всей прямой? Ответ на этот вопрос важен для нас, поскольку наш аналог непрерывного базиса Фурье в простейшем нетривиальном эллиптическом случае состоит в точности из сингулярных собственных функций Эрмита этого оператора. 
Хотелось бы напомнить, что все формальные собственные функции этого оператора были найдены Эрмитом 150 лет назад. В современной терминологии это собственные функции Блоха-Флоке. Однако почти все они имеют особенности на вещественной прямой и, тем самым, не связаны со спектральной задачей в обычном гильбертовом пространстве. В работе Эрмита использовались только те из них, которые принадлежат дискретному спектру на конечном интервале $[0, T]$ между парой соседних особенностей. Именно эти функции нужны в задаче Ламе. В то же время, никакой интерпретации сингулярных собственных функций в терминах спектральной задачи на всей прямой известно не было.

В нашей работе мы строим индефинитное скалярное произведение, естественно связанное с нашей задачей ${ }^{1}$. Это наш основной результат, являющийся частью более общей конструкции; мы строим индефинитное скалярное произведение по функциям Бейкера-Ахиезера несамосопряженных "алгебраических" периодических операторов, собственные функции Блоха-Флоке которых мероморфны на римановых поверхностях конечного рода (так же, как и в гладкой конечнозонной теории).

В нашей работе мы предполагаем, что все конечнозонные операторы периодичны по вещественной переменной $x \in \mathbb{R}$. По-видимому, основные результаты работы верны и для общих квазипериодических по $x$ конечнозонных потенциалов, однако их обобщение на квазипериодический случай может потребовать преодоления дополнительных технических трудностей.

ЗАмечАниЕ 1. Сингулярные собственные функции Блоха-Флоке естественно возникают в теории $(k+1)$-частичного оператора Калоджеро-Мозера с эллиптическим потенциалом Вейерштрасса в случае, когда константа связи равна $n(n+1)$. Они образуют (в предположении, что центр масс неподвижен) $k$-мерное алгебраическое многообразие. Аналогов результата Эрмита для $k>1$ до настоящего времени получено не было: ни одна из построенных до настоящего времени собственных функций не является собственной функцией дискретного спектра в области, ограниченной особенностями. Наш случай отвечает $k=1$. Нам представляется весьма вероятным, что эти алгебраические семейства собственных функций "обслуживают" спектральную задачу в некотором индефинитном скалярном произведении на пространстве функций на всем $\mathbb{R}^{k}$, подобно тому что мы имеем для $k=1$.

\section{1. Канонические контуры и скалярные произведения функций Бейкера-Ахиезера}

Пусть заданы неособая алгебраическая кривая (риманова поверхность) $Г$ с отмеченной точкой $P=\infty \in \Gamma$ и локальная координата $z=k^{-1}$ в окрестности $P$ такая, что $z(P)=0$. Пусть также зафиксирован “дивизор" полюсов $D=\gamma_{1}+\cdots+\gamma_{g}$ на $Г$. Построим стандартную функцию Бейкера-Ахиезе-

\footnotetext{
${ }^{1}$ Индефинитное скалярное произведение векторов состояний в гильбертовом пространстве возникало в физической литературе: для модели Ли квантовой теории поля [7]-[10], теории бозонной струны при $d=26$ ("тахион"). В последние годы К. Бендер [11] стал использовать его для одномерного оператора Шрёдингера в некоторых случаях, далеких от изучаемых нами. Некоторые аспекты абстрактной теории рассматривались и в математической литературе [12]-[14].
} 
ра (БА) $\Psi_{D}(x, z), z \in \Gamma$, мероморфную по переменной $z$, с полюсами первого порядка в точках $\gamma_{j} \in \Gamma$ и асимптотикой $\Psi=\exp \{i k x\}\left(1+O\left(k^{-1}\right)\right)$. Определим $d \mu$ как дифференциальную 1-форму с асимптотикой $d \mu=d k+$ регулярные члены в окрестности бесконечной точки $P$ и нулями в точках дивизора $D$. Множество нулей $d \mu$ имеет вид $(d \mu)=D+D^{*}$, причем

$$
D+D^{*} \sim K+2 P .
$$

В этой формуле означает так называемую “линейную эквивалентность" дивизоров в алгебраической геометрии, $K$ - дивизор дифференциальных форм. Тем самым, дивизор $D^{*}$ полностью определяется дивизором $D$. Двойственная функция и двойственная 1-форма Бейкера-Ахиезера были введены И. М. Кричевером много лет назад. Они активно использовались в совместных работах [1]-[3]. Двойственная функция имеет асимптотику $\Psi_{D}^{*}(x, z)=\exp \{-i k x\} \times$ $\left(1+O\left(k^{-1}\right)\right)$ в точке $P$, ее дивизор равен $D^{*}$. Двойственная 1-форма Байкера-Ахиезера имеет вид $\Psi^{*} d \mu$. Для скалярной двойственной функции БейкераАхиезера имеет место соотношение

$$
\Psi_{D}^{*}(x, z)=\Psi_{D^{*}}(-x, z) .
$$

Обе функции $\Psi_{D}(x, z), \Psi_{D}^{*}(x, z)$ мероморфны по $x$.

Канонические контуры $\kappa_{c}$ задаются уравнением $p_{\mathrm{I}}=c$, где $d p$ - мероморфный дифференциал второго рода такой, что $d p=d k+$ регулярные члены вблизи точки $P=\infty$ и интегралы от него по любому замкнутому контуру, не проходящему через точку $P$, чисто вещественны: $\oint_{\gamma} d p \in \mathbb{R}$. Мнимая часть $p$, которую мы обозначаем $p_{\text {I }}$ уже однозначна. Посколъку контур $\kappa_{c}$ задан как линия уровня однозначной вещественной функции на ориентированном многообразии, то он автоматически ориентирован. Специальный канонический контур отвечает выбору с $=0$, при этом константу интегрирования для р выбираем так, чтобы выражение $\exp \{i k x\}$ было ограничено для всех вещественнъх $x \in \mathbb{R}$ $u k \rightarrow \infty$ вдоль этого контура.

ЗАмЕчАниЕ 2. Конечнозонные операторы, построенные по кривой Г, периодичны по $x$ с периодом $T$ тогда и только тогда, когда $e^{i T p}$ однозначны на $\Gamma$, или, что эквивалентно, для $d p$ имеет место:

$$
\oint_{s} d p=\frac{2 \pi}{T} n_{s}, \quad n_{s} \in \mathbb{Z},
$$

где $s$ - произвольный замкнутый контур.

ЗАмЕчАниЕ 3. В работе авторов [4] особое внимание было уделено случаю, когда все точки дивизора $D$ расположены в бесконечности: $D=g P=g \infty$, где $g$ - род $Г$. Для этого случая авторами было установлено следующее свойство мультипликативности “базиса Фурье" из функций Бейкера-Ахиезера:

$$
\Psi_{g \infty}(x, z) \Psi_{g \infty}(y, z)=L_{g} \Psi_{g \infty}(x+y, z) .
$$

В этой формуле $L$ - линейный дифференциальный оператор по переменной $x$, коэффициенты которого не зависят от $z \in \Gamma, L_{g}=\partial_{x}^{g}+\cdots$ (см. [4]). Эта конструкция обобщает конструкцию работ [1]-[3] дискретных базисов Фурье на римановых поверхностях, предложенную в конце 1980-х и использованную в теории замкнутых бозонных струн. Отметим, что в нелинейных задачах (таких 
как теория струн) мультипликативные свойства базисов Фурье крайне важны. С точки зрения линейного гармонического анализа, развиваемого в данной работе, этот специальный случай существенно не отличается от других обсуждаемых потенциалов. Поскольку функция $\Psi$ обязательно имеет полюсы при вещественных значениях $x$, скалярное произведение оказывается индефинитным (см. лемму 3). Более эффективные явные формулы для коэффициентов $L_{g}$ были получены в работе [15] (см. также [16]).

Введем $\mathbb{C}$-линейное скалярное произведение на гладких функциях, заданных на контурах $\kappa_{c} \subset \Gamma$. Оно зависит от выбора дивизора $D$ и порождается базисом функций $\Psi_{D}(x, z)$, ограниченных на канонические контуры $\kappa_{c}$.

УтвеРЖДЕНИЕ 1. Для базисных функций Бейкера-Ахиезера имеет место соотношение ортогональности на римановой поверхности (на контурах $\left.\kappa_{c} \subset \Gamma\right)$ :

$$
\left(\Psi_{D}(x, z), \Psi_{D}(y, z)\right)_{\kappa_{c}}=\int_{\kappa_{c}} \Psi_{D}(x, z) \Psi_{D^{*}}(-y, z) d \mu(z)=2 \pi \delta(x-y) .
$$

Точный смысл этого утверждения и определение $\delta$-функции зависят от того, для каких функциональных классов это скалярное произведение определено. Для нестационарных самосопряженных операторов с положительным скалярным произведением это соотношение было получено в работе [17] (см. там формулу (12)). Мъ будем использовать его для сингулярных операторов, когда скалярное произведение индефинитно. Наиболее важен для нас случай специальных канонических контуров $c=0$. Приведем набросок доказательства для случая, когда $x$ и $y-$ регулярные точки.

Форма в правой части уравнения голоморфна по переменной $z$, поэтому интеграл не зависит от $c$. При $x>y$ интеграл стремится к нулю при $c \rightarrow+\infty$, и, следовательно, он равен нулю. Аналогично, если $x<y$, то равенство нулю получается устремлением $c$ к $-\infty$. Тем самым,

$$
\left(\Psi_{D}(x, z), \Psi_{D}(y, z)\right)_{\kappa_{c}}=0 \quad \text { при } x \neq y .
$$

Если мы меняем подынтегральное выражение вне окрестности точки $P$, то результат интегрирования меняется на регулярную функцию. Разлагая функции $\Psi_{D}(x, z), \Psi_{D}^{*}(x, z)$ в окрестности точки $P$ :

$$
\begin{aligned}
& \Psi_{D}(x, z)=e^{i k x}\left[1+\frac{\phi(x)}{k}+O\left(\frac{1}{k^{2}}\right)\right], \\
& \Psi_{D}^{*}(x, z)=e^{-i k x}\left[1+\frac{\phi^{*}(x)}{k}+O\left(\frac{1}{k^{2}}\right)\right],
\end{aligned}
$$

получаем:

$$
\begin{aligned}
& \left(\Psi_{D}(x, z), \Psi_{D}(y, z)\right)_{\kappa_{c}} \\
& \quad=\int_{-\infty}^{+\infty} e^{i k(x-y)}\left[1+\frac{\phi(x)+\phi^{*}(y)}{k}\right] d x+\text { регулярная функция } \\
& \quad=2 \pi \delta(x-y)+\pi i \operatorname{sgn}(x-y)\left[\phi(x)+\phi^{*}(y)\right]+\text { регулярная функция. }
\end{aligned}
$$

Форма $\Psi_{D}(x, z) \Psi_{D}^{*}(x, z) d \mu(z)$ мероморфна на $\Gamma$ с единственным полюсом в точке $P$, следовательно, его вычет равен 0 и $\phi(x)+\phi^{*}(y)=0$ при $x=y$.

Сопоставление с (2) завершает доказательство. 
Рассмотрим класс функций $\phi(z)$ на специальном каноническом контуре $\kappa_{0}$ таких, что их БА-преобразование Фуръе корректно определено. Мы будем их интерпретировать как БА-Фуръе-компоненты функции $\tilde{\phi}(x)$ в нашем $Б A$-базисе Фуръе $\Psi_{D^{*}}(-x, z)$, используя интеграл:

$$
\tilde{\phi}(x)=(\sqrt{2 \pi})^{-1} \int_{\kappa_{c}} \phi(z) \Psi_{D^{*}}(-x, z) d \mu .
$$

УТВЕРЖДЕНИЕ 2. Поскольку функиии Бейкера-Ахиезера ограничены на контуре $\kappa_{0}$ вблизи точки $P=\infty$, то данный интеграл сходится вблизи $\infty$, ecлu $\phi(k)=o\left(k^{-1+\varepsilon}\right), \varepsilon>0$.

Обратное БА-преобразование Фуръе дается формулой

$$
\phi(z)=(\sqrt{2 \pi})^{-1} \int \tilde{\phi}(x) \Psi_{D}(x, z) d x .
$$

Если функции Бейкера-Ахиезера регулярны по $x$, то интеграл понимается в обычном смысле. Как будет показано ниже, после соответствующей регуляризации эта формула применима и для сингулярных операторов. При этом существенно используются свойства пространств функций переменной $x$, получающихся как образ БА-преобразования Фуръе. Эти пространства будут обсуждаться ниже для вещественного случая, после того как будут заданъ пространства функций $H_{D, \kappa_{0}}$ на соответствующем контуре, лежащем на кривой Г. Для наиболее важного гиперэллиптического случая эти конструкции приведены в приложении В.

Выше мы рассматривали функции Бейкера-Ахиезера как функции на специальном каноническом контуре, занумерованные точками вещественной прямой. В то же время функции Бейкера-Ахиезера можно рассматривать как базис в $x$-пространстве, занумерованный точками $z$ канонического контура. Если функции Бейкера-Ахиезера регулярны по $x$, то имеет место формула

$$
\left(\Psi_{D}(x, z), \Psi_{D}(x, w)\right)_{x}=\left[\int_{x} \Psi_{D}(x, z) \Psi_{D^{*}}(-x, w) d x\right] d \mu=2 \pi \delta(z, w) .
$$

Здесь предполагается, что $z, w \in \Gamma$ принадлежат одному контуру $\kappa_{c}$. В данном случае $\delta$-функция является 1 -формой по $w$ (с учетом ориентации контура $\kappa_{c}$ ). После регуляризации эта формула верна и для сингулярных потенциалов.

В данном месте мы используем предположение о периодичности по $x \in \mathbb{R}$, чтобы исключить возможность накопления полюсов вблизи бесконечности. Исследование ситуации для квазипериодических потенциалов мы считаем целесообразным отложить до будущих работ.

Случай критических контуров, отвечающих критическим значениям функции $p_{\mathrm{I}}$, требует отдельного рассмотрения.

Обсудим теперь, для каких классов функций БА-преобразование Фурье корректно определено. Пространства функций, возникающие при этом, существенно зависят от дивизора $D$ и геометрии контура $\kappa_{c}$. В частности важно: Имеют ли функиии Бейкера-Ахиезера особенности при вещественных $x$ ? Содержит ли дивизор бесконечные точки? Является ли наш контур критическим? 
Обсуждение последнего вопроса мы отложим.

Для функций Бейкера-Ахиезера с полюсами введем следующее правило: при интегрировании по переменной $x \in \mathbb{R}$ мы обходим все полюсы в верхней полуплоскости $x+i \varepsilon, \varepsilon>0$. Корректность этой регуляризации обеспечивается следующей леммой.

Лемма 1. Пусть $z, w$ - произвольная пара конечных точек кривой Г. Тогда произведение функиий Бейкера-Ахиезера $\Psi_{D}(x, z) \Psi_{D^{*}}(-x, w)$ мероморфно по переменной $x$ и его вычеты во всех полюсах равны 0.

Доказательство леммы сразу следует из леммы 7 и леммы 4, доказанных ниже. Фактически приложение А посвящено строгому доказательству этого утверждения для полюсов произвольного порядка. Для полюсов первого порядка оно сразу следует из локального представления функций Бейкера-Ахиезера вблизи особенности: $\Psi(x, z)=\alpha /\left(x-x_{0}\right)+O\left(x-x_{0}\right)$.

Равенство нулю всех вычетов означает, что определенные указанным выше образом интегралы по переменной $x \in \mathbb{R}$ не зависят от того, с какой стороны мы обходим полюсы.

Вещественные алгебраические кривые. Предположим теперь, что наши данные обратной задачи, состоящие из кривой $\Gamma$ с отмеченной точкой $P=\infty$ и локальным параметром $z=k^{-1}$ вблизи $P$, вещественны. Это означает, что на данных обратной задачи действует антиголоморфная инволюция

$$
\tau: \Gamma \rightarrow \Gamma, \quad \tau^{2}=1,
$$

такая, что $\tau(P)=P$ и $\tau^{*}(k)=\bar{k}$.

Дифференциал $d p$ в этом случае удовлетворяет соотношению $\tau^{*}(d p)=d \bar{p}$. Выберем константу интегрирования для $p$ так, чтобы имело место соотношение $\tau^{*}\left(p_{\mathrm{I}}\right)=-p_{\mathrm{I}}$. Тогда линия уровня $\kappa_{0}=\left(p_{\mathrm{I}}=0\right)$ инвариантна относительно $\tau$ :

$$
\tau: \kappa_{0} \rightarrow \kappa_{0}
$$

и дифференциалы $d k, d p$ вещественны на $\kappa_{0}$.

Отметим, что контур $\kappa_{0}$ содержит все неподвижные точки $\tau$ :

$$
\operatorname{Fix}_{\tau} \subset \kappa_{0},
$$

где $z \in \operatorname{Fix}_{\tau}$ означает, что $\tau(z)=z$. Следующая несложная геометрическая лемма полезна, чтобы связать наши конструкции с некоторыми результатами работ конца 1980-х годов (см. [18]), посвященных описанию спектральных данных, порождающих гладкие вещественные решения уравнения КПІ с оператором Лакса $i \partial_{x}+\partial_{y}^{2}+u(x, y)$.

Лемма 2. Множество неподвижных точек Fix $_{\tau}$ антиголоморфной инволючии $\tau$ совпадает с каноническим контуром $\kappa_{0}$, если и толъко если $\mathrm{Fix}_{\tau}$ разделяет Г на две компоненты: $\Gamma=\Gamma_{+} \cup \Gamma_{-}$.

Доказательство сразу следует из очевидного замечания, что $\kappa_{0}$ всегда разделяет $\Gamma$, но никакое меньшее подмножество $\kappa_{0}$ разделяющим уже не является. Мы предполагаем, что $P \in \mathrm{Fix}_{\tau}$.

Условия вещественности на дивизор имеют вид: $\tau(D)=D^{*}$, или, что эквивалентно,

$$
D+\tau(D) \sim K+2 P
$$


где $K$ - дивизор дифференциальных форм. В этом случае $\tau^{*}(d \mu)=d \bar{\mu}$, и мы можем определить эрмитово (полуторалинейное, невырожденное, но не обязательно положительно определенное) скалярное произведение для базисных функций Бейкера-Ахиезера на контуре $\kappa_{0}$ формулой

$$
\begin{aligned}
\left\langle\Psi_{D}(x, z), \Psi_{D}(y, z)\right\rangle_{\kappa_{0}} & =\left(\Psi_{D}(x, z), \bar{\Psi}_{\tau D}(y, \tau z)\right)_{\kappa_{0}} \\
& =\int_{\kappa_{0}} \Psi_{D}(x, z) \bar{\Psi}_{D}(y, \tau z) d \mu(z) .
\end{aligned}
$$

Мы предполагаем, что интеграл вычисляется с учетом ориентации контура $\kappa_{0}$.

В этих формулах мы учитываем, что функция $\bar{\Psi}_{D}(y, \tau z)$ мероморфна по переменной $z$, имеет полюсы в точках $\tau D$ и асимптотику $\exp \{-i k y\} \times$ $\left(1+O\left(k^{-1}+\cdots\right)\right)$ вблизи $P$ для $y, k \in \mathbb{R}$. Тем самым, для вещественных переменных наше скалярное произведение совпадает с $\mathbb{C}$-линейным выражением, выписанным выше (см. (1)).

В $x$-пространстве имеется следующее скалярное произведение на базисных функциях Бейкера-Ахиезера:

$$
\left\langle\Psi_{D}(x, z), \Psi_{D}(x, w)\right\rangle_{x}=\int_{\mathbb{R}}\left[\Psi_{D}(x, z) \bar{\Psi}_{D}(\bar{x}, w) d \mu\right] d x .
$$

Отметим, что

$$
\bar{\Psi}_{D}(\bar{x}, w)=\Psi_{\tau D}(-x, \tau w)
$$

при всех вещественных $x$. Подынтегральное выражение мероморфно по $x$. Вычеты во всех полюсах по $x$ для $\Psi_{D}(x, z) \bar{\Psi}_{D}(\bar{x}, \tau z)$ равны нулю, поскольку это произведение совпадает с фигурирующим в формулировке леммы 1.

Мы приходим к следующему выводу.

Лемма 3. 1) Эрмитово скалярное произведение на контуре $\kappa_{0}$, определенное в (4), положительно определено, если и только если $\kappa_{0}=$ Fix $_{\tau} u$, кроме того, форма $d \mu$ всюду положительна на контуре $\kappa_{0}$.

2) Эрмитово скалярное произведение в х-пространстве корректно определено соглашением, что все полюсы по х обходятся в верхней полуплоскости. Оно положительно тогда и только тогда, когда наша функция Бейкера-Ахиезера $\Psi_{D}(x, z)$ не имеет полюсов при вещественных $x$.

Первая часть леммы имеет смысл, поскольку форма $d \mu$ вещественна на контуре $\operatorname{Fix}_{\tau}$. Мы имеем $\tau(z)=z$ для $z \in \operatorname{Fix}_{\tau}$, и верхняя компонента $\Gamma^{+}$кривой $\Gamma$ задает ориентацию контура $\kappa_{0}$. Условия первой части возникали в работе [18] как условия регулярности решений КПI. Вторая часть очень важна для нас, поэтому мы приводим в приложении полное доказательство, использующее так называемое ядро Коши-Бейкера-Ахиезера. Эта величина заимствована из работы [5], однако нам требуется некоторое усиление результатов этой работы. Кроме того, в работе [5] отсутствуют полные доказательства.

В результате мы имеем два возможных источника неположительности скалярного произведения на контуре $\kappa_{0}$.

1) $\kappa_{0} \neq \operatorname{Fix}_{\tau}$. В этом случае $\tau(z) \neq z$ для $z$, лежащих вне множества неподвижных точек. Скалярное произведение становится нелокальным, а значит, и неположительным. 
2) $\operatorname{Fix}_{\tau}=\kappa_{0}$, но вещественный дивизор $D$ выбран так, что знаки $d \mu$ различны на разных компонентах (см. раздел 2).

Единственным источником неположительности скалярного произведения в $x$-пространстве являются полюсы функиии $\Psi$ по переменной $x$ на вещественной оси. Это - центральное наблюдение нашей работы.

Более детальному обсуждению наиболее важного гиперэллиптического случая посвящен следующий раздел.

\section{2. Индефинитные скалярные произведения на гиперэллиптических римановых поверхностях. Операторы Шрёдингера с сингулярными потенциалами}

Сконцентрируемся теперь на наиболее интересном для нас классе гиперэллиптических римановых поверхностей Г, отвечающих периодическим операторам второго порядка $L$. Пусть $\Gamma$ задана в следующей форме:

$$
w^{2}=\left(u-u_{0}\right) \cdots\left(u-u_{2 g}\right)=R(u) .
$$

Точка общего положения (точка, не являющаяся точкой ветвления) записывается как пара $\gamma=(z, \pm)$. Точка ветвления $P$, отвечающая $u=\infty$, является "бесконечностью" нашей задачи, локальная координата в окрестности нее имеет вид $k^{-1}=u^{-1 / 2}=z$. Дивизор общего положения $D=\gamma_{1}+\cdots+\gamma_{g}$ определяет функцию Бейкера-Ахиезера $\Psi_{D}(x, z)$ с описанными выше аналитическими свойствами. Она является решением уравнения

$$
L \Psi=\left(-\partial_{x}^{2}+U(x)\right) \Psi(x, z)=u(z) \Psi(x, z) .
$$

Дополнительно мы предполагаем, что потенциал $U(x)$ периодичен: $U(x+T)=$ $U(x)$ для вещественных $x$. В теории конечнозонных потенциалов хорошо известны необходимые и достаточные условия того, чтобы функция $U(x)$ была вещественной и неособой (мы будем называть их каноническими данными обратной задачи), они состоят из двух частей:

1) Сильное условие вещественности на $\Gamma$ : все точки ветвления $u_{j}$ вещественны и попарно различны. Будем считать, что они пронумерованы в порядке возрастания: $u_{0}<u_{1}<\cdots<u_{2 g}$.

2) Дивизор $D$ является правилъным, т.е. все точки $D$ имеют вид $\gamma_{k}=$ $\left(\alpha_{k},+\right)$ или $\gamma_{k}=\left(\alpha_{k},-\right)$, где все $\alpha_{k}$ вещественны и удовлетворяют соотношениям: $u_{2 k-1} \leqslant \alpha_{k} \leqslant u_{2 k}, k=1, \ldots, g$. Тем самым, каждая лакуна (или, что эквивалентно, каждый $a$-цикл) содержит ровно одну точку дивизора.

На римановой поверхности $\Gamma$ действует пара коммутирующих антиголоморфных инволюций $\tau_{ \pm}: \tau_{ \pm}(u, w)=(\bar{u}, \pm \bar{w})$. Обозначим $\tau_{-}=\sigma, \tau_{+}=\tau$. Специальный канонический контур $\kappa_{0}$ является в точности множеством неподвижных точек $\tau$ : $\kappa_{0}=\mathrm{Fix}_{\tau}$, его проекция на плоскость переменной $u$ совпадает с объединением всех зон спектра.

Множество неподвижных точек $\mathrm{Fix}_{\sigma}$ инволюции $\sigma$ является объединением $g+1$ овала, его проекция на плоскость переменной $u$ совпадает с объединением всех запрещенных зон $\left(-\infty, u_{0}\right],\left[u_{2 k-1}, u_{2 k}\right], k=1, \ldots, g$. 
В обоих случаях спектр понимается как спектр оператора $L$ в гильбертовом пространстве $L^{2}(\mathbb{R})$ квадратично интегрируемых вещественных или комплекснозначных функций на вещественной оси.

Прообразы конечных запрещенных зон (лакун) - $a$-циклы $a_{k}$ на кривой $\Gamma$.

Мы имеем $\sigma(D)=D$ и $\tau D=D^{*}$, где $D+D^{*}=K+2 P$.

Опишем теперь спектральные данные, порождающие вещественные, но не обязательно регулярные операторы $L$ (неканонические вещественные данные обратной задачи):

1) кривая $Г$ является вещественной, т. е. набор точек ветвления $u_{j}, j=$ $0,1, \ldots, u_{2 k}$, инвариантен относительно антиголоморфной инволюции $u \rightarrow \bar{u}$;

2) дивизор $D$ удовлетворяет соотношению $\sigma(D)=D$, но не обязательно такой, как в каноническом случае.

Если указанные условия выполнены, но данные не являются каноническими, потенциал $U(x)$ вещественен, однако имеет полюсы при вещественных $x$. В противном случае он был бы самосопряженным в обычном положительном гильбертовом пространстве $L$, а это не так. Тем самым для всех неканонических вещественных спектральных данных оператор $L$ сингулярен.

Назовем спектральные данные вещественными полуканоническими, если спектральная кривая Г удовлетворяет сильному условию вещественности, однако дивизор не является правильным. В частности, контур $\kappa_{0}$ совпадает с множеством $\operatorname{Fix}_{\tau}$ неподвижных точек инволюции $\tau$. В этом случае потенциал $U(x)$ имеет полюсы на вещественной прямой, однако спектр оператора остается вещественным.

На контуре $\kappa_{0}$ определена естественная ориентация, поскольку он является границей ориентированной области $p_{\mathrm{I}} \geqslant 0$. Для таких кривых имеет место формула

$$
d p=\frac{\left(u-p_{1}\right) \cdots\left(u-p_{g}\right) d u}{2 \sqrt{\left(u-u_{0}\right) \cdots\left(u-u_{2 g}\right)}},
$$

где все $p_{k}$ вещественны и $p_{k} \in\left[u_{2 k-1}, u_{2 k}\right]$.

Пусть не все точки ветвления $Г$ вещественны, а среди них есть комплексно сопряженные пары. В этом случае множество $\mathrm{Fix}_{\tau}$ существенно меньше, чем контур $\kappa_{0}$. Оператор $L$ в этом случае всегда сингулярен. Он имеет комплексный спектр, совпадающий с проекцией контура $\kappa_{0}$ на плоскость комплексной переменной $u$.

В обоих случаях операторы симметричны относительно индефинитного скалярного произведения, определенного в разделе 1.

Учитывая сказанное выше, получаем следующую теорему.

Теорема 1. 1) Пусть риманова поверхность Г и дивизор D вещественнь u, кроме того, дивизор не содержит точки $P=\infty$. Тогда форма

$$
d \mu=\frac{\left(u-\gamma_{1}\right) \cdots\left(u-\gamma_{g}\right) d u}{2 \sqrt{R(u)}}, \quad u=z^{-1 / 2},
$$

вещественна и отлична от нуля.

2) Проекция контура $\kappa_{0}$ на плоскость переменной и является спектром оператора $L$ в некотором пространстве $\widetilde{H}_{L}$ функиий переменной $x$, зависящем 
от полюсов оператора L (описание этого пространства для случая общего положения приведено ниже).

3) Обратное БА-преобразование Фуръе задает изометрическое отображение пространства $\widetilde{H}_{L}$ в пространство функций $H_{D, \kappa_{0}}$ наконтуре $\kappa_{0}$, и обратно. Скалярное произведение в $H_{D, \kappa_{0}}$ задается формулой

$$
\langle a, b\rangle_{\kappa_{0}}=\int_{\kappa_{0}} a(z) \bar{b}(\tau z) d \mu(z) .
$$

Оно индефинитно, если $\tau z \neq z$ для каких-либо точек контура $\kappa_{0}$ или если $\tau z=z$ и $d \mu$ вещественно, но имеет различные знаки на различных компоненmax контура.

Скорость убывания на бесконечной компоненте была обсуждена выше.

4) Если, кроме того, риманова поверхность Г удовлетворяет сильному условию вещественности (все точки ветвления вещественны), то $\tau z=z$ и знак $d \mu$ постоянен на каждой из компонент $\kappa_{0}$. В этом случае

$$
\langle a, b\rangle_{\kappa_{0}}=\int_{\kappa_{0}} a(z) \bar{b}(z) d \mu(z)
$$

и пространство $H_{D, \kappa_{0}}$ изоморфно прямой сумме обычных пространств функций на компонентах $\kappa_{0}$, взятых со знаками, задаваемыми знаком $d \mu$ с учетом ориентации на контуре $\kappa_{0}$.

5) Линейный оператор $L=-\partial_{x}^{2}+U(x)$ симметричен в пространстве $\widetilde{H}_{L}$.

6) Если часть точек дивизора $D$ лежит в бесконечности: $\gamma_{g}=\infty$, то форма $d \mu$ голоморфна.

ЗАмечАниЕ 4. Из формулы (5) следует, что знак $d \mu$ на вещественных овалах $\tau$ относительно ориентации на контуре $\kappa_{0}$ совпадает со знаком функции $d p / d \mu$, или, что эквивалентно, со знаком следующего отношения:

$$
\frac{\left(u-p_{1}\right) \cdots\left(u-p_{g}\right)}{\left(u-\alpha_{1}\right) \cdots\left(u-\alpha_{g}\right)}
$$

ЗАмечАниЕ 5. Как будет видно ниже, мы не строим точных пополнений пространств $\widetilde{H}_{L}$ и $H_{D, \kappa}$. Тем самым, наши результаты не полны с точки зрения современного функционального анализа.

ЗАмЕчаниЕ 6 . Если $r$ дивизоров $D$ лежат в точке $\infty$ (т. е. $D=r \infty+\left(\alpha_{1}, \pm\right)+$ $\left.\cdots+\left(\alpha_{g-r}, \pm\right)\right)$, то

$$
d \mu=\frac{\left(u-\alpha_{1}\right) \cdots\left(u-\alpha_{g-r}\right) d u}{2 \sqrt{R(u)}} .
$$

$B$ частном случае $r=g$ весь дивизор сосредоточен в точке $\infty$. Именно этот случай мы рассматриваем как правильный аналог обыкновенного преобразования Фуръе, поскольку именно для этой конфигурации функиии БейкераАхиезера обладают замечательными мультипликативными свойствами.

Доказательство теоремы немедленно следует из результатов раздела 1 и приложения А. 
2.1. Описание индефинитного пространства $\widetilde{H}_{L}$ для вещественных сингулярных конечнозонных $x$-периодических операторов $L$ общего положения. Пусть $U(x)$ является вещественным сингулярным конечнозонным $x$-периодическим потенциалом с периодом $T$, причем полюсы на окружности $[0, T]$ занумерованы в циклическом порядке: $x_{0}=0<x_{1}<\cdots<x_{k}<T$. Особенности таких потенциалов имеют вид

$$
U=\frac{n(n+1)}{\left(x-x_{j}\right)^{2}}+O(1) .
$$

Для потенциалов общего положения

$$
U=\frac{2}{\left(x-x_{j}\right)^{2}}+O(1)
$$

вблизи каждого из полюсов $x_{j}$. Рассмотрим пространство функций на вещественной прямой $f(x) \in \widetilde{H}_{x_{0}, \ldots, x_{k}}$, зависящее только от положения полюсов $x_{j}$ и задаваемое условием: вблизи каждой из особенностей имеет место представление

$$
f(x)=\frac{\alpha_{j}}{x-x_{j}}+O\left(x-x_{j}\right)
$$

где разность $f(x)-\alpha_{j} /\left(x-x_{j}\right)=O\left(x-x_{j}\right)$ является $C^{\infty}$-гладкой вблизи точки $x=x_{j}$. Легко видеть, что оператор $L=-\partial^{2}+U(x)$ отображает это пространство в себя:

$$
L f(x) \in \widetilde{H}_{x_{0}, \ldots, x_{k}} .
$$

Пространство $\widetilde{H}_{x_{0}, \ldots, x_{k}}$ естественно представляется как прямой интеграл пространств $\widetilde{H}_{x_{0}, \ldots, x_{k}, \varkappa}$ по окружности $|\varkappa|=1$. Пространство $\widetilde{H}_{x_{0}, \ldots, x_{k}, \varkappa}$ определяется как подпространство в $\widetilde{H}_{x_{0}, \ldots, x_{k}}$, выделяемое условием Блоха-Флоке:

$$
f(x+T)=\varkappa f(x) .
$$

Функции Бейкера-Ахиезера (Блоха-Флоке) для всех $z \in \kappa_{0}$ принадлежат $\widetilde{H}_{x_{0}, \ldots, x_{k}, \varkappa}$ для некоторого $\varkappa$. Это следует из формул для них, полученных в теории конечнозонных операторов.

Eстественно поставитъ вопрос: связаны ли вычеты для сингулярных линейных операторов какими-нибудь (зависящими от $L$ ) нетривиальными линейными соотношениями? В случае, если такие соотношения существуют, нужно выбрать линейное подпространство $\mathbb{C}^{q}(L, \varkappa) \subset \mathbb{C}^{k}$, в котором вычеты принимают значения; оно должно зависеть как от оператора $L$, так и от мультипликатора Блоха-Флоке $\varkappa$. Его размерность $q=q(L)$ равна числу отрицательных квадратов в нашем скалярном произведении при фиксированном $\varkappa$. Случай высших потенциалов Ламе обсуждается в приложении В. Тем самым,

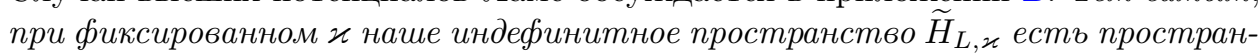
ство $F_{x_{0}, \ldots, x_{k}}(\varkappa)$ функций $f$ с мультипликатором $\varkappa$ и нулями первого порядка во всех полюсах $x_{j}$ плюс конечномерное пространство $\mathbb{C}^{q}(L, \varkappa) \subset \mathbb{C}^{k}$ :

$$
\widetilde{H}_{L, \varkappa}=\mathbb{C}^{q}(L, \varkappa) \oplus F_{x_{0}, \ldots, x_{k}}(\varkappa) .
$$

Полное пространство $\widetilde{H}_{L} \subset \widetilde{H}_{x_{0}, \ldots, x_{k}}$ представляется как прямой интеграл пространств $\widetilde{H}_{L, \varkappa}$ по окружности. 
Во многих случаях удается доказать, что $k=q$, т.е. между вычетами нет соотношений. Это означает, в частности, что пространство $\widetilde{H}_{L}$ полностью определяется полюсами (см. приложение В). Согласно нашему предположению, это верно для всех конечнозонных операторов с полюсами общего положения. Естественное расширение на периодические потенциалы с изолированными полюсами вида $n(n+1) / x^{2}+O(x)$ обсуждается ниже в примере 1 (cmp. 65).

Отметим, что мероморфность собственных функций $\Psi \sim \alpha /\left(x-x_{j}\right)^{n}, n \in \mathbb{Z}$ автоматически влечет сингулярность потенциала вида $n(n+1) /\left(x-x_{j}\right)^{2}+O(1)$.

Рассмотрим произвольную функцию $a(z)$ на контуре $\kappa_{0}$, достаточно быстро убывающую при $z \rightarrow 0$ или $k \rightarrow \infty$. Легко убедиться, что ее БА-преобразование Фурье

$$
f(x)=\tilde{a}(x)=\frac{1}{\sqrt{2 \pi}} \oint_{\kappa_{0}} a(z) \Psi_{D^{*}}(x, z) d \mu
$$

принадлежит к описанному выше классу с достаточно хорошим убыванием при $|x| \rightarrow \infty$. Мы обсудим глобальное поведение и пополнение пространства в приложении $\mathrm{B}$, используя операторы на окружности $[0, T]$ с периодическими (квазипериодическими) граничными условиями на концах с равными 1 по модулю мультипликаторами Блоха-Флоке $\varkappa,|\varkappa|=|\exp \{i p(z) T\}|=1$, отвечающими контуру $\kappa_{0}$, на котором $p_{\mathrm{I}}=0$.

2.2. Примеры: случай $g=1$. Мы обсудим следующие два случая.

Первый пример. Оператор Эрмита-Ламе. Рассмотрим вещественную кривую Г рода $g=1$ с вещественными точками ветвления $u_{0}, u_{1}, u_{2}, \infty$. Тем самым, это вещественный полуканонический случай. Мы будем предполагать, что дивизор $D=\gamma$ совпадает с точкой $P=\infty$. Функция Бейкера-Ахиезера $\Psi_{D}(x, z)$ была найдена Эрмитом как базис решений для всех значений спектрального параметра $u(z)=z^{-2}$. В данном случае $L$ - оператор Ламе; его потенциал $U(x)=2 \wp(x)$ периодичен и имеет полюсы вида $2 / x^{2}$ на вещественной прямой по $x$ в точках $n T, n \in \mathbb{Z}$. Ученые XIX века рассматривали этот оператор на интервале $[0, T]$. Они получили этот оператор как результат разделения переменных в координатах Якоби для оператора Лапласа-Бельтрами на трехосном эллипсоиде. Им был нужен спектр на интервале $[0, T]$ с нулевыми граничными условиями на концах. В литературе обычно используется термин "спектр Дирихле", однако для конечнозонных операторов мы будем использовать термин "спектр Эрмита".

Обсудим полезную с методологической точки зрения связь спектра Эрмита (Дирихле) с периодическим спектром неособого сдвинутого периодического оператора $L_{i \omega}$ на окружности $[0, T]$ и спектром на всей прямой $\mathbb{R}$.

УтВЕРЖДЕНИЕ 3. Эрмитов спектр оператора Ламе L прост. Он состоит в точности из точек $\lambda_{s}, s \in \mathbb{Z}^{+}$, являюшихся дважды вырожденными собственными значениями сдвинутого периодического оператора $L_{i \omega}$ на окружности. Здесъ $2 i \omega$ - мнимый период функции ю(x).

Хорошо известно, что потенциал $2 \wp(x+i \omega)$ играет важную роль в теории солитонов: эта функция задает движущиеся волны (“солитонные решетки") 
в теории уравнения КдФ, открытые в XIX веке Кортевегом и де Фризом. Рассмотрим соответствующий оператор Лакса

$$
L_{i \omega}=-\partial_{x}^{2}+2 \wp(x+i \omega) .
$$

Теория периодической задачи для уравнения КдФ началась в 1974 г. с "солитонного вывода и понимания" спектра этого оператора. В частности, ее невырожденные собственные значения для периодических или антипериодических граничных условий

$$
L_{i \omega} \psi(x)=\lambda \psi(x), \quad \psi(x+T)= \pm \psi(x)
$$

в точности совпадают с точками ветвления $u_{0}, u_{1}, u_{2}$ римановой поверхности $\Gamma$. Эта задача имеет бесконечно много вырожденных собственных значений $\lambda_{s}$ с $u>u_{2}$ и теми же граничными условиями. Это в точности набор эрмитовых собственных значений для несдвинутого оператора $L$. Они совпадают с экстремальными точками функции $S(u)$, которая является половиной следа матрицы монодромии $\widehat{T}(u)$ :

$$
S(u)=\frac{1}{2} \operatorname{Tr} \widehat{T}, \quad \widehat{T}(\psi(x, u))=\psi(x+T, u)
$$

для минимального периода $T$. Мы имеем $-1 \leqslant S(u) \leqslant 1$ для $u \in\left[u_{2}, \infty\right]$ и $S(u)= \pm 1, S^{\prime}(u)=0$ для $u=\lambda_{s}$. В частности, $\lambda_{s}>u_{2}$.

ДокАЗАтЕльство. Задача Эрмита вещественна, поэтому мы будем искать вещественные собственные функции $\psi\left(x, \lambda_{s}\right)=\psi_{s}(x)$, paвные 0 на концах интервала $[0, T]$. Пространство неособых решений $L \psi=\lambda_{s} \psi$ не более чем одномерно, следовательно, оно должно быть собственным вектором матрицы монодромии $\widehat{T}$ с вещественным собственным значением. Поэтому это либо дважды вырожденная точка, упомянутая в формулировке, либо точка запрещенной зоны. Как мы уже отмечали, все функции Бейкера-Ахиезера оператора Ламе имеют полюсы в точках $n T$. Утверждение доказано.

В точках $u=\lambda_{s}$ матрица монодромии $\widehat{T}$ равна \pm 1 . Одна из собственных функций неособа и равна $n T$, являясь тем самым собственной функцией эрмитова спектра. Поэтому он полностью определяется римановой поверхностью Г.

Вернемся к спектральной теории оператора Ламе $L$ на всей прямой $x \in \mathbb{R}$ и вещественном каноническом контуре $z \in \kappa_{0}$.

Пространство функций $H_{D, \kappa_{0}}$ представляется как прямая сумма двух пространств:

$$
H_{D, \kappa_{0}}=H_{0} \oplus H_{\infty}
$$

Здесь $H_{0}$ - функции на компактном цикле $c_{1} \subset \Gamma$, расположенном над спектральной зоной $\left[u_{0}, u_{1}\right]$ (конечной зоной спектра). Второе подпространство $H_{\infty}$ состоит из функций на $\mathbb{R} \subset \Gamma$ лежащем над $\left[u_{2}, \infty\right]$ и гомеоморфном $\mathbb{R}=S^{1} \backslash \infty$. Эти функции должны убывать на бесконечности, как указано в разделе 1.

УтВЕРЖДЕНИЕ 4. Индефинитное скалярное произведение на пространстве $H_{\infty, \kappa_{0}}=H_{0} \oplus H_{\infty}$ положительно на $H_{\infty}$ и отрицательно на $H_{0}$.

ДокАЗАТЕЛЬСтво. В этом случае $d \mu=d z / \sqrt{4\left(u-u_{0}\right)\left(u-u_{1}\right)\left(u-u_{2}\right)}$ и ориентация контура $\kappa_{0}=c_{1} \cup c_{\infty}$ такова, что $\left.d \mu\right|_{c_{1}}<0,\left.d \mu\right|_{c_{\infty}}>0$. Утверждение доказано. 
Полезно сравнить эту ситуацию с самосопряженным случаем, когда $\gamma^{\prime}=$ $\left(\alpha^{\prime}, \pm\right)$ и $\alpha^{\prime} \in\left[u_{1}, u_{2}\right]$ расположено в лакуне. Тогда

$$
d \mu^{\prime}=\frac{\left(u-\alpha^{\prime}\right) d u}{\sqrt{4\left(u-u_{0}\right)\left(u-u_{1}\right)\left(u-u_{2}\right)}},
$$

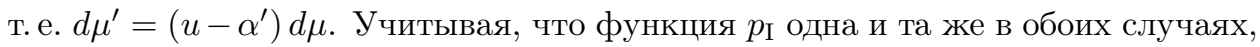
получаем, что множитель $\left(u-\alpha^{\prime}\right)$ имеет противоположные знаки на $c_{1}$ и $c_{\infty}$. Тем самым скалярное произведение положительно, как и должно быть.

Второй пример. Комплексные точки ветвления. Пусть $g=1$, но точки ветвления $Г$ имеют вид $u_{0}=\bar{u}_{1} \in \mathbb{C}, u_{3} \in \mathbb{R}$. Этот случай является вещественным, но не полуканоническим. Точка дивизора лежит, как и раньше, в бесконечности: $D=\infty$. Соответствующий потенциал также вещественен: $U(x)=2 \wp(x)$, имеет полюс $2 / x^{2}$ и вещественный период $T$, но решетка периодов имеет ромбическую структуру (комплексные периоды не ортогональны вещественному, но их длины равны). Контур $\kappa_{0}$ связен и является критическим. Он состоит из пары окружностей, трансверсально пересекающих друг друга в паре вещественных точек $\left(u^{*}, \pm\right)$ на бесконечной компоненте $\left[u_{2}, \infty\right]$. Тем самым спектр оператора $L=-\partial_{x}^{2}+2 \wp(x)$ на всей прямой, обсуждающийся в данной работе, содержит невещественную часть, на которой антиголоморфная инволюция $\tau$ действует нетривиально: $\operatorname{Fix}_{\tau} \neq \kappa_{0}$. Эрмитов спектр этой задачи корректно определен (в полюсах $[0, T]$ накладываются нулевые граничные условия). Он совпадает с множеством вещественных точек, для которых $S(u)= \pm 1$, где $S=(1 / 2) \operatorname{Tr} \widehat{T}$, a $\operatorname{Tr} \widehat{T}$ - след матрицы монодромии (вещественный для вещественных $x$ ). Все эти точки расположены внутри спектральной зоны $\left[u_{2}, \infty\right]$.

Обычное преобразование Фуръе отвечает роду 0: риманова поверхность $Г$ имеет две точки ветвления $u_{0}=0, u_{1}=\infty$. Спектральная зона на $\Gamma$ состоит из одной компоненты $c_{\infty}$, расположенной над $[0, \infty]$. Она изоморфна $\mathbb{R}=S^{1} \backslash \infty$, мера $d \mu$ совпадает со стандартной. Тем самым, наше пространство - в точности стандартное $H=H_{\infty}=L^{2}(\mathbb{R})$, и скалярное произведение положительно. Для рода большего 0 скалярное произведение положительно лишь для гладких потенциалов с точками дивизора, расположенными в лакунах (ровно по одной в каждой из лакун).

Вернемся к общим сингулярным конечнозонным потенциалам. Во всех случаях, когда дивизор содержит бесконечную точку или точку в бесконечной запрещенной зоне, мы имеем индефинитное скалярное произведение. В этом случае сдвигом по времени всегда можно переместить хотя бы одну из точек дивизора в бесконечность.

Во всех случаях, когда одна из лакун содержит более одной точки дивизора или хотя бы одна из точек дивизора лежит вне запрещенных зон (например комплексна), мы также имеем индефинитное скалярное произведение.

Знак скалярного произведения, отвечающего циклу $c_{j} \subset \Gamma$, расположенному над зоной $\left[u_{2 j-1}, u_{2 j}\right]$, легко определяется.

Пусть даны точки дивизора $\gamma_{s}=\left(\alpha_{s}, \pm\right)$, где $\alpha_{s} \in\left[u_{2 q_{s}-1}, u_{2 q_{s}}\right], s=1, \ldots, r$, и $\gamma_{r+k}=\infty$ при всех $k>r$. Как мы уже знаем, знак скалярного произведения 
совпадает со знаком $d p / d \mu$ на цикле $c_{k}$, где

$$
d \mu=\frac{\left(u-\alpha_{1}\right) \cdots\left(u-\alpha_{r}\right) d z}{\sqrt{4\left(u-u_{0}\right) \cdots\left(u-u_{2 g}\right)}},
$$

причем ориентация $\kappa_{0}$ задается функцией $p_{\text {I }}$, как это было объяснено в разделе 1. Например, при $r=0$ (когда мы имеем мультипликативность БА-преобразования Фуръе, основанного на потенциалах Эрмита-Ламе $n(n+1) \wp(x)=$ $U(x))$ знаки скалярного прочзведения на контурах $c_{k}$ образуют знакопеременную последовательность. В этом случае

$$
d \mu=\frac{d u}{2 \sqrt{R(u)}} .
$$

Его мы обсудим подробнее в приложении В.

\section{Приложение А. Ядро Коши-Бейкера-Ахиезера}

В работе [5] было введено понятие ядра Коши-Бейкера-Ахиезера. При этом неявно предполагалось, что спектральные данные порождают несингулярные (возможно, комплексные) потенциалы. Убедимся, что основные свойства этого ядра верны и в сингулярном случае.

Напомним, что ядро Коши-Бейкера-Ахиезера $\omega(x, z, w)$ определяется как функция трех переменных $x \in \mathbb{C}, z \in \Gamma \backslash P, w \in \Gamma \backslash P$ со следующими аналитическими свойствами.

1) При фиксированном $x$ ядро $\omega(x, z, w)$ является мероморфной функцией переменной $z$ и мероморфной 1-формой переменной $w$.

2) При фиксированных $x, w$ ядро $\omega(x, z, w)$ имеет ровно $g+1$ простой полюс по $z$ в точках $\gamma_{1}, \ldots, \gamma_{g}, w$.

3) При фиксированных $x, z$ ядро $\omega(x, z, w)$ имеет простые нули $w$ в точках $\gamma_{1}, \ldots, \gamma_{g}$ и простой полюс с вычетом 1 в точке $z$. В локальных координатах имеем:

$$
\omega(x, z, w)=\frac{d w}{w-z}+\text { (регулярная функция) } \quad \text { при } w \rightarrow z .
$$

4) При фиксированных $x, w$ ядро $\omega(x, z, w)$ имеет существенную особенность по $z$ в точке $P=\infty$ :

$$
\omega(x, z, w)=e^{i k(z) x} \cdot O\left(\frac{1}{k(z)}\right) .
$$

$5)$ при фиксированных $x, z$ ядро $\omega(x, z, w)$ имеет существенную особенность по $w$ в точке $P=\infty$ :

$$
\omega(x, z, w)=\left[e^{-i k(w) x} \cdot O\left(\frac{1}{k(w)}\right)\right] d k(w) .
$$

Для спектральных данных общего положения ядро Коши-Бейкера-Ахиезера $\omega(x, z, w)$ существует и единственно. Это легко доказать по той же схеме, по которой доказывается существование и единственность функции БейкераАхиезера с использованием теоремы Римана-Роха. По аналогии с [5] докажем следующую лемму. 
Лемма 4. Имеет место следующая формула:

$$
\partial_{x} \omega(x, z, w)=-i \Psi(x, z) \Psi^{*}(x, w) d \mu(w) .
$$

ДокАЗАТЕЛЬСтво. При фиксированном $w$ левая часть (7) имеет следующие свойства:

1) $\partial_{x} \omega(x, z, w)$ мероморфна по переменной $z$ на $\Gamma \backslash P$ и имеет ровно $g$ простых полюсов в точках $\gamma_{1}, \ldots, \gamma_{g}$;

2) $\partial_{x} \omega(x, z, w)=O(1) e^{i k(z) x}$ при $z \rightarrow P$.

Тем самым, при фиксированном $w$ выражение $\partial_{x} \omega(x, z, w)$ пропорционально $\Psi(x, z)$. Аналогично, при фиксированном $z$ выражение $\partial_{x} \omega(x, z, w)$ пропорционально $\Psi^{*}(x, w) d \mu(w)$. Тем самым,

$$
\partial_{x} \omega(x, z, w)=c \Psi(x, z) \Psi^{*}(x, w) d \mu(w) .
$$

Устремляя одновременно $z$ и $w$ к точке $P$, мы получаем, что $c=-i$. Лемма доказана.

ЗАмЕчАНИЕ 7 . Пусть $x=0$. В этом случае $\omega(0, z, w)$ совпадает со стандартным мероморфным аналогом ядра Коши на замкнутых римановых поверхностях, введенным в [19], [20].

ЗАмЕЧАнИЕ 8 . Естественно ввести ядро $\omega(\vec{t}, z, w)$, зависящее от всех времен уравнения Кадомцева-Петвиашвили $\vec{t}=\left(t_{1}, t_{2}, t_{3}, \ldots\right), x=t_{1}, y=t_{2}, t=t_{3}$ (см. [5]). Существенная особенность ядра $\omega(\vec{t}, z, w)$ имеет следующий вид:

$$
\begin{aligned}
& \omega(\vec{t}, z, w)=\exp \left\{i \sum_{j=1}^{\infty} t_{j} k^{j}(z)\right\} \cdot O\left(\frac{1}{k(z)}\right), \quad z \rightarrow P, \\
& \omega(\vec{t}, z, w)=\exp \left\{-i \sum_{j=1}^{\infty} t_{j} k^{j}(w)\right\} \cdot O\left(\frac{1}{k(w)}\right) d k(w), \quad w \rightarrow P .
\end{aligned}
$$

(Мы предполагаем, что лишь конечное число времен $t_{n}$ отлично от 0.)

Чтобы подчеркнуть зависимость ядра $\omega(\vec{t}, z, w)$ от дивизора $D=\gamma_{1}+\cdots+\gamma_{g}$, мы будем при необходимости писать $\omega_{D}(\vec{t}, z, w)$. Имеет место следующая несложная лемма.

ЛЕмма 5. Обозначим $D(\vec{t})$ дивизор нулей функции $\Psi_{D}(\vec{t}, z)$. Тогда при любом $\vec{t}_{*}$ мы имеем следующее соотношение:

$$
\omega_{D}(\vec{t}, z, w)=\frac{\Psi_{D}\left(\vec{t}_{*}, z\right)}{\Psi_{D}\left(\vec{t}_{*}, w\right)} \omega_{D\left(\vec{t}_{*}\right)}\left(\vec{t}-\vec{t}_{*}, z, w\right) .
$$

Для нас особенно важен следующий частный случай, порождающий сингулярные операторы.

Лемма 6. Предположим, что ровно одна точка дивизора $D$ лежит в бесконечно удаленной точке $P=\infty: D=\gamma_{1}+\gamma_{2}+\cdots+\gamma_{g-1}+P$. Тогда для набора точек общего вида $\gamma_{1}, \ldots, \gamma_{g-1}$ имеет место особенно простая формула 
для ядра $\omega(\vec{t}, z, w)$ :

$$
\begin{aligned}
\omega(\vec{t}, z, w) & =\frac{\theta\left[\sum_{j} \vec{U}_{j} t_{j}+\vec{A}(z)-\vec{A}(w)-\vec{A}\left(\gamma_{1}\right)-\cdots-\vec{A}\left(\gamma_{g-1}\right)-\vec{K}\right]}{\theta\left[\sum_{j} \vec{U}_{j} t_{j}-\vec{A}\left(\gamma_{1}\right)-\cdots-\vec{A}\left(\gamma_{g-1}\right)-\vec{K}\right]} \\
& \times \frac{C d \mu(w)}{\theta\left[\vec{A}(z)-\vec{A}(w)-\vec{A}\left(\gamma_{1}\right)-\cdots-\vec{A}\left(\gamma_{g-1}\right)-\vec{K}\right]} \exp \left[i \sum_{j} t_{j} \int_{w}^{z} \Omega_{j}\right] .
\end{aligned}
$$

$B$ этой формуле $\Omega_{j}$ - мероморфные дифференциалы с единственным полюсом в точке $P$ :

$$
\Omega_{j}=d\left(k^{j}\right)+\text { регулярная часть }
$$

и нулевыми а-периодами, $U_{j}$ - нормализованный вектор b-периодов дифференчиала $\Omega_{j}$ :

$$
U_{j}^{k}=\frac{1}{2 \pi} \oint_{b_{k}} \Omega_{j}
$$

$\vec{A}(\gamma)$ - преобразование Абеля с начальной точкой интегрирования $P, \vec{K}$-вектор римановых констант, $d \mu$ - голоморфный дифференциал с нулями в точках $\gamma_{1}, \ldots, \gamma_{g-1}$. Пусть $z$ - локальная координата в точке $P$ такая, что $d \mu=d z(1+o(1))$. Тогда нормализующая константа $C$ задается следующей формулой:

$$
C=\left.\partial_{\nu}\right|_{\nu=0} \theta\left[-\vec{A}(v)-\vec{A}\left(\gamma_{1}\right)-\cdots-\vec{A}\left(\gamma_{g-1}\right)-\vec{K}\right] .
$$

ДокАзАтЕльство. Несложный стандартный анализ показывает, что выражение (13) однозначно по обеим переменным $z$ и $w$ на кривой Г и для вектора $\vec{t}$ общего положения имеет полюсы и нули по $z$ и $w$ в нужных точках. Пусть $z \rightarrow w$. Тогда

$$
\omega(\vec{t}, z, w) \sim \frac{C d \mu(w)}{\theta\left[\vec{A}(z)-\vec{A}(w)-\vec{A}\left(\gamma_{1}\right)-\cdots-\vec{A}\left(\gamma_{g-1}\right)-\vec{K}\right]} .
$$

Если $z$ отлично от $\gamma_{1}, \ldots, \gamma_{g}, \gamma_{1}^{*}, \ldots, \gamma_{g}^{*}$, то знаменатель в (15) имеет нуль первого порядка по $w$ при $w=z$. Если $z=\gamma_{j}, j=1, \ldots, g$, то знаменатель тождественно обращается в нуль. Если же $z=\gamma_{j}^{*}, j=1, \ldots, g$, то знаменатель имеет нуль второго порядка. Тем самым, нули дифференциала знаменателя по $w$ при $w=z$ совпадают с нулями числителя и вычет (15) на диагонали $w=z-$ регулярная функция на Г. Тем самым, вычет (15) на диагонали - константа. Нормализация (14) в точности означает, что вычет стремится к 1 при $z \rightarrow \infty$, что и завершает доказательство.

Из (13) немедленно следует, что ядро Коши-Бейкера-Ахиезера мероморфно по всем переменным $t_{j}$. Сопоставляя (13) с (12), мы получаем следующий результат.

Лемма 7. Для произвольного дивизора $D$ такого, что $\Psi_{D}(x, z)$ определена при почти всех $x$, ядро $\omega_{D}(x, z, w)$ мероморфно по $x$.

ЗАмЕчАниЕ 9. Пусть операторы, построенные по кривой Г, периодичны по $x$ с периодом $T$. Тогда имеет место следующая формула:

$$
\omega(x+T, z, w)=\omega(x, z, w) e^{i[p(z)-p(w)] x} .
$$


Выведем соотношения ортогональности (3) для функций Бейкера-Ахиезера, рассматриваемых как функции переменной $x$ со спектральным параметром $z$. В регулярном случае это скалярное произведение было исследовано в работе [13], оно использовалось позднее так же в работе [21]. Прямое вычисление с учетом (9) дает:

$$
\begin{aligned}
\int_{-n T}^{n T} & \Psi(x, z)\left[\Psi^{*}(x, w) d \mu\right] d x=\left.i \omega(x, z, w)\right|_{-n T} ^{n T}= \\
= & i \omega(0, z, w)\left[e^{i[p(z)-p(w)] n T}-e^{-i[p(z)-p(w)] n T}\right] \\
= & {[p(w)-p(z)] \omega(0, z, w) \int_{-n T}^{n T} e^{i[p(z)-p(w)] x} d x }
\end{aligned}
$$

Тем самым,

$$
\lim _{n \rightarrow \infty} \int_{-n T}^{n T} \Psi(x, z)\left[\Psi^{*}(x, w) d \mu\right] d x=2 \pi[p(w)-p(z)] \omega(0, z, w) \delta(p(z)-p(w)),
$$

следовательно,

$$
\lim _{n \rightarrow \infty} \int_{-n T}^{n T} \Psi(x, z)\left[\Psi^{*}(x, w) d \mu\right] d x=0 \quad \text { при } z \neq w, z, w \in \kappa_{c} .
$$

Пусть $w \rightarrow z$. Используя (6) и учитывая то, что ориентация специального канонического контура $\kappa_{c}$ определяется формой $d p$, мы получаем окончательный ответ: Для бункиий Бейкера-Ахиезера, рассматриваемых как базис в х-пространстве, имеют место следующие соотношения:

$$
\begin{aligned}
& \left(\int_{-\infty}^{\infty} \Psi(x, z) \Psi^{*}(x, w) d x\right) d \mu \\
& =2 \pi(p(w)-p(z))\left[\frac{d p(w)}{p(w)-p(z)}+\text { регулярные члены }\right] \delta(p(z)-p(w))=2 \pi \delta(z, w) .
\end{aligned}
$$

Здесь мы подразумеваем, что используется стандартное соглашение об обходе сингулярностей на вещественной прямой.

\section{Приложение В. Гиперэллиптический случай. Периодические граничные условия}

В.1. Ядро Коши-Бейкера-Ахиезера. На гиперэллиптических кривых ядро Коши-Бейкера-Ахиезера задается простой явной формулой, вывод которой находится на элементарном учебном уровне:

$$
\omega(x, z, w)=i \frac{\Psi(x, z) \Psi_{x}^{*}(x, w)-\Psi_{x}(x, z) \Psi^{*}(x, w)}{w-z} d \mu(w) .
$$


Чтобы доказать ее, достаточно проверить, что для правой части выполнены все аналитические свойства, определяющие это ядро. Кроме того, легко вычисляется ее производная по $x$ :

$$
\begin{aligned}
\partial_{x} \omega & (x, z, w)=\left[\Psi(x, z) \Psi_{x x}^{*}(x, w)-\Psi_{x x}(x, z) \Psi^{*}(x, w)\right] \frac{i}{w-z} d \mu \\
& =\left[(-z-U(x)) \Psi(x, z) \Psi^{*}(x, w)-(-w-U(w)) \Psi(x, z) \Psi^{*}(x, w)\right] \frac{i}{w-z} d \mu \\
& =-i \Psi(x, z) \Psi^{*}(x, w) d \mu .
\end{aligned}
$$

В.2. Задача с периодическими граничными условиями. Пусть наш конечнозонный оператор периодичен с периодом $T$. В этом случае пространство $L^{2}(\mathbb{R})$ функций на всей прямой естественно разложить в прямой интеграл пространств функций $H(\varkappa)$ с мультипликатором $|\varkappa|=1$ :

$$
\begin{gathered}
H(\varkappa)=\{f(x): f(x+T)=\varkappa f(x)\}, \quad|\varkappa|=1 . \\
L^{2}(\mathbb{R})=\int_{|\varkappa|=1}^{\oplus} H(\varkappa) .
\end{gathered}
$$

Соответственно параллельно со спектральной задачей на всей прямой естественно рассматривать задачи с фиксированным мультипликатором, равным по модулю 1:

$$
\Psi(x+T, z)=\varkappa \Psi(x, z), \quad|\varkappa|=1 .
$$

Для вещественных регулярных потенциалов эти задачи самосопряжены и их спектр дискретен. Отметим все точки $z_{j}$ на кривой $\Gamma, j=1,2, \ldots, \infty$, такие, что

$$
e^{i T p\left(z_{j}\right)}=\varkappa
$$

Все они лежат на специальном каноническом контуре $\kappa_{0}$, причем каждый из конечных овалов содержит лишь конечное число точек $z_{j}$.

ЛЕмма 8. Скалярное произведение базисных функиий дается формулой:

$$
\int_{0}^{T} \Psi\left(x, z_{j}\right) \Psi^{*}\left(x, z_{k}\right) d x=\delta_{j k} \frac{d p\left(z_{j}\right)}{d \mu\left(z_{j}\right)} .
$$

Как и ранъше, мъ сдвигаем контур в х-плоскости, чтобы обойти сингулярности.

Отметим, что для регулярного самосопряженного случая эта формула хорошо известна - см. работу [17], формула (30). Наша лемма обобщает этот результат на случай сингулярных потенциалов и индефинитных скалярных произведений.

ДокАЗАТЕЛЬСтво. При $j \neq k$

$$
\int_{0}^{T} \Psi\left(x, z_{j}\right) \Psi^{*}\left(x, z_{k}\right) d x=\left.\frac{i \omega\left(x, z_{j}, z_{k}\right)}{d \mu\left(z_{k}\right)}\right|_{0} ^{T}=\frac{i \omega\left(0, z_{j}, z_{k}\right)}{d \mu\left(z_{k}\right)}\left[e^{i T\left[p\left(z_{j}\right)-p\left(z_{k}\right)\right]}-1\right]=0 .
$$


Пусть теперь $k=j$ :

$$
\begin{aligned}
\int_{0}^{T} & \Psi\left(x, z_{j}\right) \Psi^{*}\left(x, z_{j}\right) d x=\left.\lim _{w \rightarrow z_{j}} \frac{i \omega\left(x, z_{j}, w\right)}{d \mu(w)}\right|_{0} ^{T} \\
& =\lim _{w \rightarrow z_{j}}\left[\frac{i d p(w)}{\left[p(w)-p\left(z_{j}\right)\right] d \mu(w)}+\text { регулярные члены }\right]\left[e^{i T\left[p\left(z_{j}\right)-p(w)\right]}-1\right] \\
& =\frac{d p\left(z_{j}\right)}{d \mu\left(z_{j}\right)} .
\end{aligned}
$$

Предположим теперь, для "спектральных данных" $\Gamma, D$ выполнены условия вещественности. Учитывая, что для вещественных потенциалов $\Psi^{*}(x, z)=$ $\bar{\Psi}(x, \tau z)$, мы получаем следующую теорему.

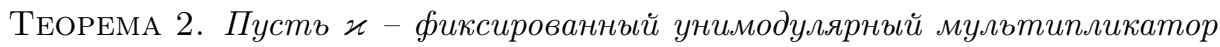
такой, что $|\varkappa|=1$. Зададим скалярное произведение формулой

$$
\left(\Psi\left(x, z_{j}\right), \Psi\left(x, z_{k}\right)\right)_{x}=\int_{0}^{T} \Psi\left(x, z_{j}\right) \bar{\Psi}\left(\bar{x}, z_{k}\right) d x .
$$

Для сингулярных потенциалов оно индебинитно, причем число отрицательных квадратов равно числу точек $z_{j}$ таких, что $d p\left(z_{j}\right) / d \mu\left(z_{j}\right)<0$. Тем самым мы получаем пространства Понтрягина-Соболева, на которых наши операторы симметричны.

Пример 1 (высшие потенциалы Ламе). Рассмотрим операторы $L_{n}=-\partial^{2}+$ $n(n+1) \wp(x)$ (полуканонические в нашей терминологии) с эллиптической функцией, определенной на римановой поверхности: $y^{2}=\left(u-u_{0}\right)\left(u-u_{1}\right) \times$ $\left(u-u_{2}\right), u_{j} \in \mathbb{R}$. Случай $n=1$ обсуждался в разделе 2 . При всех $n \in \mathbb{Z}^{+}$ эти потенциалы - особые конечнозонные. Они отвечают некоторым алгебраическим кривым $\Gamma_{n}$ рода $g=n$ и имеют полюсы в точках $m T, m \in \mathbb{Z}$. Здесь $T-$ вещественный период функции $\wp(x)$. Оператор $L_{n, i \omega}=-\partial^{2}+n(n+1) \wp(x+i \omega)$ вещественен и регулярен, если $i \omega$ - мнимый полупериод. Он имеет ту же спектральную кривую $\Gamma_{n}$.

Напомним, что эрмитов спектр $L_{n}$ определяется как набор уровней энергии, для которых существует собственная функция оператора $L_{n}$, обращающаяся в 0 на обоих концах интервала $[0, T]$. Несложно проверяется, что эрмитов спектр задается уравнениями $S_{n}(u)= \pm 1, S_{n}^{\prime}(u)=0$. Здесь $S_{n}=$ $(1 / 2) \operatorname{Tr} \widehat{T}_{n}$, где $\operatorname{Tr} \widehat{T}_{n}-$ след оператора монодромии для оператора $L_{n}$ (или $L_{n, i \omega}$, поскольку их спектральные кривые совпадают). Дивизор оператора $L_{n}$ имеет вид $D=n \infty$. Для оператора $L_{n, i \omega}$ с четным неособым потенциалом дивизор расположен в правых концах лакун: $u_{n, 2 k}^{*}, k=1, \ldots, n$, алгебраической кривой $\Gamma_{n}: w^{2}=\left(u-u_{0}\right) \cdots\left(u-u_{2 g}\right)$. Как и выше, мы предполагаем, что концы зон занумерованы в порядке возрастания: $u_{0}<u_{1}<\cdots<u_{2 g}$. Так же как и для $n=1$, весь эрмитов спектр $L_{n}$ расположен в бесконечной спектральной зоне $\left[u_{n, 2 n}^{*}, \infty\right]$ (см. обсуждение случая $n=1$ в разделе 2 ).

Обсудим конструкцию пространства $\widetilde{H}_{L}$ для оператора $L=L_{n}$. Элементарный анализ лорановских разложений вблизи точки $x=0$ приводит к следующему выводу. Собственные функции оператора $L_{n}$ nри всех энергиях имеют 
следующую структуру:

$$
\begin{aligned}
n=2 k-1: & \psi(x)=\frac{\alpha_{1}}{x^{n}}+\frac{\alpha_{2}}{x^{n-2}}+\cdots+\frac{\alpha_{k}}{x}+\phi(x), \\
n=2 k: & \psi(x)=\frac{\alpha_{1}}{x^{n}}+\frac{\alpha_{2}}{x^{n-2}}+\cdots+\frac{\alpha_{k}}{x^{2}}+\phi(x),
\end{aligned}
$$

где $\phi(x)$ - регулярная функция такая, что

$$
\phi(0)=\cdots=\phi^{(2 r)}(0)=\cdots=\phi^{(2 k-2)}(0)=0 \quad \text { при } n=2 k-1
$$

И

$$
\phi^{\prime}(0)=\cdots=\phi^{(2 r+1)}(0)=\cdots=\phi^{(2 k-1)}(0) \quad \text { при } n=2 k^{2}
$$

Понятно, что любые линейные комбинации собственных функций также удовлетворяют указанным линейным соотношениям. Тем самым мы приходим к следующему заключению.

Рассмотрим пространство функиий класса $C^{\infty}$ с унимодулярнъмм мулътипликатором $|\varkappa|=1: f(x) \in F_{n, \varkappa}, f(x+T)=\varkappa f(x)$ и следующими условиями в точке 0:

$$
\begin{aligned}
& f(x)-f(-x)=O\left(x^{n}\right), \quad n=2 k, \\
& f(x)+f(-x)=O\left(x^{n}\right), \quad n=2 k-1 .
\end{aligned}
$$

Зададим на этих пространствах стандартное положительное скалярное произведение. Рассмотрим пространство $F_{n, \varkappa} \oplus \mathbb{C}^{k}(\varkappa)$, где $\mathbb{C}^{k}(\varkappa)$ над $\mathbb{C}$ порождается особенностями сингулярных функиий Бейкера-Ахиезера с блоховским

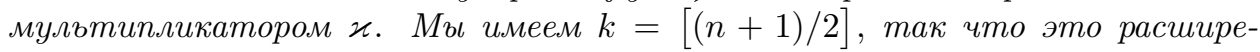
ние в точности соответствует набору "въчетов" $\alpha_{1}, \ldots, \alpha_{k}$. Все функции из указанного расширения имеют особенности, описанные выше. Прямой интеграл построенных пространств по окружности $\varkappa \in S^{1}$ дает наше пространство $\widetilde{H}_{L_{n}}$ с индебинитной метрикой.

Построим решения уравнения КдФ

$$
U_{t}=6 U U_{x}-U_{x x x}
$$

с начальными условиями $U(x, 0)=n(n+1) \wp(x)$. Оно имеет вид:

$$
U(x, t)=\sum_{j=1}^{j=n(n+1) / 2} 2 \wp\left(x-x_{j}(t)\right),
$$

причем $x_{j}(0)=0$. Мы предполагаем, что $x_{j} \neq x_{p}$ при достаточно малых $t \neq 0$.

УтвеРЖДЕНИЕ 5. При $t \rightarrow+0$ имеет место асимптотика $x_{j} \sim a_{j} t^{1 / 3}$, причем $a_{j} \neq a_{p}$. Величины $a_{j}$ - некоторые комплексные числа. Набор $a_{j}$ инвариантен относительно поворотов, кратных $2 \pi / 3: a_{j} \rightarrow \eta a_{j}$, где $\eta^{3}=1$. Каждая из орбит группы $\mathbb{Z}_{3}$, порожденной $\eta$, входит в этот набор только один раз. Действие группы свободно, если $n(n+1) / 2$ делится на 3. Если же $n(n+1) / 2=1(\bmod 3)$, то существует ровно одна нулевая орбита: $a_{n(n+1) / 2}=0$. Остальные орбиты попарно различны $\left(\right.$ m.е. $a_{j}^{3} \neq a_{k}^{3}$, если они принадлежат разным орбитам).

\footnotetext{
2 Данные рассеяния для сингулярных потенциалов с особенностями обсуждаемого вида с $n=1$ были исследованы в работе [22]. Индефинитные скалярные произведения в [22] не обсуждались.
} 
Доказательство этого утверждения использует несложное наблюдение, что при малых $t$ асимптотика полюсов эллиптического решения аппроксимируется асимптотикой полюсов рационального решения: $U_{0}=\sum_{j} 2 /\left(x-x_{j}(t)\right)^{2}$ с начальным условием $x_{j}(0)=0$. Для каждого из полюсов мы имеем:

$$
\partial_{t} x_{j}(t)=-12 \sum_{p \neq j} \frac{1}{\left(x_{j}-x_{p}\right)^{2}} .
$$

Сделаем замену времени, чтобы исключить несущественный множитель -12 . При этом новое время мы будем обозначать той же самой буквой $t$. Подставляя $x_{j}=a_{j} t^{1 / 3}$ в уравнение $(21)$ и пользуясь тем, что (19) должно выполняться тождественно по $t$, мы получаем систему алгебраических соотношений на $a_{j}^{3}$, из которых и следует наше утверждение.

ГиПотеЗА. Число “вещественных" орбит $\left(a_{j}^{3} \in \mathbb{R}\right)$ в точности равно $k=$ $[(n+1) / 2]$.

Отметим, что рациональные решения иерархии КдФ с начальными условиями $U_{0}(x, 0)=n(n+1) / x^{2}$ были исследованы в работе [23]. Для этих решений были получены явные формулы. В частности, в этой работе было доказано, что по крайней мере один полюс всегда вещественен, поскольку соответствующий оператор Лакса не является самосопряженным.

В соответствии с результатами настоящей статьи, $k=[(n+1) / 2]$ в точности равно числу отрицательных квадратов в неположительном скалярном произведении для всех значений $\varkappa$.

УТВеРЖДЕНИЕ 6. Для числа вещественных орбит имеем оценку $k^{\prime} \geqslant k=$ $[(n+1) / 2]$.

Противоположное неравенство требует доказательства. Наше пространство $\widetilde{H}_{L_{t}}$ для оператора $L_{t}=-\partial_{x}^{2}+U(x, t)$ получается как расширение пространства функций класса $C^{\infty}$ с нулями в вещественных точках $x_{j}(t)$. Тем самым, размерность сингулярного слагаемого равна числу полюсов, если и только если отсутствуют линейные соотношения между вычетами. Размерность отрицательного подпространства для $L_{t}$ стабильна относительно деформаций из иерархии КдФ (не зависит от $t$ ).

Данная гипотеза была численно проверена нами для $n<9$. В частности:

при $n=1$ мы имеем: $a_{1}=0$;

при $n=2$ мы имеем $a_{j}=\left(1, \eta, \eta^{2}\right)$;

при $n=3$ мы имеем: $a_{j}=\left(a, a \eta, a \eta^{2}, b, b \eta, b \eta^{2}\right)$; здесь

$$
w=\frac{a^{3}}{b^{3}}=\frac{1}{2}(-7+\sqrt{45})
$$

и обе орбиты вещественны:

$$
a^{3}=1+\frac{9 w(w+2)}{(w-1)^{2}}, \quad b^{3}=1+\frac{9 w^{-1}\left(w^{-1}+2\right)}{\left(w^{-1}-1\right)^{2}} .
$$

В качестве следствия мы получаем следующий важный результат. $B$ данном частном случае нет соотношении между вычетами в вещественных особых точках конечнозонных вещественных сингулярных потенииалов общего положения. Тем самым, пространство $\widetilde{H}_{L_{t}}$ бункций переменной $x$ для $L=-\partial_{x}^{2}+U(x, t)$ зависит только от положения вещественных полюсов. 


\section{В.3. Обобщения: промежуточные и однополюсные конечнозонные сингулярные потенциалы.}

Промежуточные потенциалы. Рассмотрим произвольную неособую гиперэллиптическую кривую $\Gamma$, заданную уравнением $y^{2}=\left(u-u_{0}\right) \cdots\left(u-u_{2 n}\right)$ с вещественными точками ветвления $u_{0}<\cdots<u_{2 n}$. Пусть дивизор имеет вид $D=r \infty+\gamma_{1}+\cdots+\gamma_{n-r}$, причем все конечные точки $\gamma_{j}=\left(\lambda_{j}^{*}, \pm\right)$ лежат в конечных лакунах $a_{k}=\left[u_{2 k-1}, u_{2 k}\right], k \geqslant 1$ (не более одной точки в каждой из лакун). Предположим дополнительно, что потенциал периодичен по вещественной переменной $x$ с минимальным периодом $T$. Тогда $U(x)$ имеет конечное число полюсов специального вида $r(r+1) / x^{2}+O(x)$ на окружности $[0, T]$.

Однополюсные потенциалы. Пусть наш потенциал имеет ровно один полюс на периоде в точке 0. Можно показать, что эрмитов спектр в этом случае есть объединение пары множеств: множества точек, в которых $S(u)= \pm 1$, $S^{\prime}=0$, и множества точек $\lambda_{j}^{*}$, расположенных в конечных лакунах. Эрмитов спектр, отвечающий фиксированной спектральной кривой $Г$, содержит минимально возможное число точек, если $r=n$, и максимально возможное, если $r=0$, т. е. потенциал неособ. Все промежуточные потенциалы с полюсом в точке 0 получаются из регулярного с $r=0$ применением специальной последовательности преобразований Бэклунда-Дарбу (Г при этом не меняется).

Несложно вычислить (для всех $r \leqslant n$ ) число собственных функций с отрицательными скалярными квадратами для периодического спектра. Присвоим каждой из зон спектра знак + или - . При этом мы будем двигаться справа налево (в сторону уменьшения энергии). Бесконечную зону спектра пометим знаком + . Зону слева от нее пометим знаком + , если в лакуне между ними есть точка дивизора, и знаком - , если между ними точек дивизора нет. Далее будем придерживаться правила: знаки, приписанные двум зонам, совпадают, если в лакуне между ними есть точка дивизора, и противоположны, если в лакуне точек дивизора нет. Всего мы имеем $r$ перемен знаков (заметим, что $r-$ это в точности число лакун, в которых нет дивизора). Кроме того, дополнительные собственные функции с отрицательными квадратами порождаются точками зон со знаком минус такими, что $S(u)=1, S^{\prime}=0$. Эти точки могут рассматриваться как вырожденные лакуны длины 0 с дополнительной точкой дивизора на этой лакуне, так что при прохождении через нее знак не меняется. Максимально возможное число отрицательных квадратов соответствует случаю $r=1$, когда пустая лакуна - лакуна с максимальным номером $\left[u_{2 n-1}, u_{2 n}\right]$. Детальное описание индефинитного пространства функций переменной $x$ зависит от числа полюсов, которое трудно предсказать.

В.4. Построение бесконечнозонных потенциалов с конечным $r$, равным числу пустых лакун. Этот класс потенциалов может быть особенно интересен. Можно определить бесконечнозонный предел описанной выше конструкции. А именно, будем сохранять постоянными число $r$, пустые лакуны и вещественный период $T$, а также вид и число полюсов, одновременно увеличивая род $n \rightarrow \infty$ путем открытия все большего числа малых лакун с новыми точками дивизора внутри бесконечной спектральной зоны. Мы будем открывать лакуны вблизи точек, удовлетворяющих условиям $S(u)= \pm 1$, так, чтобы их длины достаточно быстро убывали с ростом номеров. По-видимому, такие потенциалы можно построить, стартуя с гладкого потенциала и приме- 
няя специальную последовательность преобразований Бэклунда-Дарбу. Было бы интересно доказать, что все такие потенциалы аппроксимируются сингулярными конечнозонными с тем же периодом и фиксированным $r$.

B.5. Потенциалы с одним полюсом. Рассмотрим специальный потенциал такой, что $D=n \infty$, периодичный по $x$ с минимальным периодом $T$. Предположим, что соответствующий оператор (точнее, его спектральная кривая $\Gamma$ ) является малым возмущением оператора Ламе с потенциалом $n(n+1) \times$ $\wp(x)$. В этом случае потенциал $U(x)$ имеет только один вещественный полюс на окружности $[0, T]$ с особенностью $n(n+1) / x^{2}+O\left(x^{2}\right)$. Сформулированные выше для оператора Ламе результаты остаются верными и в этом случае (некоторые из них требуют, чтобы возмущенный потенциал также был четен).

Можно построить более общие потенциалы с одним полюсом. Рассмотрим произвольный гладкий конечнозонный периодический потенциал $U(x)$ с минимальным периодом $T$. Следуя [24], можно применить к $U(x)$ специальное преобразование Бэклунда (Дарбу), называемое преобразованием Крума. Результирующий потенциал $U_{1}(x)$ периодичен с тем же периодом. Он имеет ту же самую спектральную кривую Г и ровно один полюс на периоде $[0, T]$ в точке 0 с особенностью $2 / x^{2}+O(x)$. Спектр Дирихле (эрмитов спектр в нашей работе) оператора с потенциалом $U_{1}(x)$ получается из спектра Дирихле-Эрмита потенциала $U(x)=U_{0}$ исключением основного состояния. Можно повторить эту процедуру, $U_{j} \rightarrow U_{j+1}$, исключая на каждом шаге основное состояние спектра и увеличивая коэффициент при полюсе в точке 0 с $j(j+1) / x^{2}+O(x)$ до $(j+1)(j+2) / x^{2}+O(x)$.

УТВЕРЖДЕНИЕ 7. Обозначим $U_{r}(x)$ потенциал, полученный из гладкого конечнозонного потенциала $U(x)=U_{0}$ применением $r$ итераций преобразования Крума к функииям основного состояния. Тогда $U_{r}(x)$ периодичен с периодом $T$ $u$ имеет ровно один полюс на периоде в точке $x=0$ вида $r(r+1) / x^{2}+O(x)$. Спектральная кривая потенциала $U_{r}(x)$ совпадает со спектральной кривой $U(x)=U_{0}$, спектр Дирихле-Эрмита потенциала $U_{r}(x)$ получается удалением $r$ низиих точек из спектра Дирихле-Эрмита $U(x)$.

Эта процедура может быть также применена к периодическим гладким бесконечнозонным потенциалам с минимальным периодом $T$.

B.6. Примеры: вырожденные случаи. Обсудим теперь вырожденные однозонные сингулярные потенциалы, отвечающие вырождениям спектральных эллиптических кривых, порождающих эллиптические функции Вейерштрасса и операторы Ламе.

\section{а. Тригонометрические (гиперболические) вырождения.}

Пусть вещественный период стремится к бесконечности, тогда как мнимый остается конечным:

$$
2 \wp(x)+\text { const } \rightarrow \frac{2 c^{2}}{\sinh ^{2}(c x)}=U_{c}(x) .
$$

В этом случае эллиптические функции отвечают кривой $\Gamma_{t}, t \rightarrow 0$. Она является спектральной кривой соответствующего оператора с точками ветвления $u_{0}^{t}<u_{1}^{t}<u_{2}^{t}<\infty$ такими, что $u_{j}^{t} \in \mathbb{R}$. В процессе вырождения $t \rightarrow 0$ мы имеем

$$
u_{0}, u_{1} \rightarrow-c^{2}, \quad u_{2} \rightarrow 0 .
$$


Точка дивизора $\gamma$ лежит в точке $\infty$ при всех $t$. Легко убедиться, что спектр оператора $L_{c}=-\partial_{x}^{2}+U_{c}(x)$ в соответствующем пространстве $\widetilde{H}_{L_{c}}$ на всей прямой $\mathbb{R}$ занимает всю полупрямую $\lambda \geqslant 0$ и содержит одну отрицательную точку $\lambda_{0}=-c^{2}$ кратности 1 с собственной функцией

$$
\psi_{c}=\frac{1}{\sinh (c x)} .
$$

Эта собственная функция имеет отрицательный квадрат, на всех других собственных функциях скалярное произведение положительно. Тем самым, мы имеем пространство Понтрягина-Соболева с одним отрицательным квадратом.

b. Рациональное вырождение $c \rightarrow 0, U \rightarrow 2 / x^{2}$. Оба периода стремятся к бесконечности и $u_{j}^{t} \rightarrow 0, j=0,1,2$. Спектр оператора $L=-\partial_{x}^{2}+2 / x^{2}$ на всей прямой - полупрямая $\lambda \geqslant 0$. При $\lambda=0$ мы имеем сингулярную собственную функцию $\psi_{0}=1 / x$ (дискретное собственное значение на дне непрерывного спектра), скалярный квадрат которой отрицателен.

с. Несложно также построить тригонометрическое вырождение

$$
U=\frac{2 c^{2}}{\sin ^{2}(c x)}
$$

с антипериодическим собственным значением, отвечающим концу предельной “конечной спектральной зоны” $0 \leqslant \lambda$, где $\lambda \geqslant c^{2}$ :

$$
\psi_{0}=\frac{1}{\sin (c x)}, \quad L_{c} \psi_{0}=c^{2} \psi_{0} .
$$

Бесконечная спектральная зона начинается в той же точке $\lambda \geqslant c^{2}$. Здесь вещественный период остается конечным, а мнимый стремится к бесконечности. Этот случай соответствует вырождению вещественной эллиптической кривой, при котором длина конечной лакуны стремится к нулю: $u_{1}=u_{2}=c^{2}, u_{0}=0$. Как и раньше, точка дивизора лежит в $\infty$. Спектр в этом случае - полупрямая $\lambda \geqslant u_{0}$. Для каждого мультипликатора Блоха-Флоке $\varkappa \in S^{1}$ имеется ровно одна собственная функция с собственным значением $0 \leqslant \lambda_{0}(\varkappa) \leqslant c^{2}$, скалярный квадрат которой отрицателен в нашей индефинитной метрике. Основное состояние $\phi_{0}=\cos (c x) / \sin (c x), \lambda=0$ отвечает чисто периодическому случаю $\varkappa=1$. Этот потенциал получается из постоянного потенциала $U=0$ обсуждавшимся выше преобразованием Крума-Дарбу с $c=1$ :

$$
\begin{gathered}
L \rightarrow \widetilde{L}, \quad L=-\left(\partial_{x}+a\right)\left(\partial_{x}-a\right)=-\partial_{x}^{2}+U, \quad L \eta=\lambda^{\prime} \eta, \\
\widetilde{L}=-\left(\partial_{x}-a\right)\left(\partial_{x}+a\right)=-\partial_{x}^{2}+\widetilde{U}, \quad a=(\log \eta)_{x}, \\
{\left[L f=\mu f, \tilde{f}=\left(\partial_{x}-a\right) f\right] \rightarrow[\tilde{L} \tilde{f}=\mu \tilde{f}],} \\
\widetilde{U}=U-2 a_{x}=U-2(\log \eta)_{x x} .
\end{gathered}
$$

Полагая $U=0, \lambda^{\prime}=1, \eta=\sin x$, получаем

$$
\widetilde{U}=\frac{2}{\sin ^{2} x}, \quad[\mu=0, f=1] \rightarrow \tilde{f}=\phi_{0}=\frac{\cos x}{\sin x}
$$

И

$$
[\mu=1, f=\cos x] \rightarrow\left[\tilde{f}=\left(\partial_{x}-a\right) \cos x=-1 / \sin x\right]
$$




\section{Список литературы}

[1] И. М. Кричевер, С.П. Новиков, “Алгебры типа Вирасоро, римановы поверхности и структуры теории солитонов", Функи. анализ и его прил., 21:2 (1987), 46-63; англ. пер.: I. M. Krichever, S. P. Novikov, "Algebras of Virasoro type, Riemann surfaces and structures of the theory of solitons", Funct. Anal. Appl., 21:2 (1987), $126-142$.

[2] И. М. Кричевер, С. П. Новиков, "Алгебры типа Вирасоро, римановы поверхности и струны в пространстве Минковского", Функи. анализ и его прил., 21:4 (1987), 47-61; англ. пер.: I. M. Krichever, S. P. Novikov, "Virasoro-type algebras, Riemann surfaces and strings in Minkowsky space", Funct. Anal. Appl., 21:4 (1987), 294-307.

[3] И. М. Кричевер, С.П. Новиков, “Алгебры типа Вирасоро, тензор энергии-импульса и операторные разложения на римановых поверхностях", Функи. анализ и его прил., 23:1 (1989), 24-40; англ. пер.: I. M. Krichever, S. P. Novikov, "Algebras of Virasoro type, energy-momentum tensor, and decomposition operators on Riemann surfaces", Funct. Anal. Appl., 23:1 (1989), 19-33.

[4] P. G. Grinevich, S. P. Novikov, "Topological charge of the real periodic finite-gap sine-Gordon solutions", Comm. Pure Appl. Math., 56:7 (2003), 956-978.

[5] P. G. Grinevich, A. Yu. Orlov, "Virasoro action on Riemann surfaces, Grassmannians, $\operatorname{det} \bar{\partial}_{J}$ and Segal-Wilson $\tau$-function", Problems of modern quantum field theory (Alushta, 1989), Springer, Berlin, 1989, 86-106.

[6] Г. Бейтман, А. Эрдейи, Высшие трансцендентные функции. Эллиптические и автоморфные функиии. Функиии Ламе и Матъе, Наука, М., 1967; пер. с англ.: A. Erdélyi, W. Magnus, F. Oberhettinger, F. G. Tricomi, Higher transcendental functions. Vol. III, McGraw-Hill, New York-Toronto-London, 1955 (based, in part, on notes left by H. Bateman).

[7] T. D. Lee, "Some special examples in renormalizable field theory", Phys. Rev. (2), 95:5 (1954), 1329-1334.

[8] G. Källén, W. Pauli, "On mathematical structure of T. D. Lee's model of a renormalizable field theory", Danske Vid. Selsk. Mat.-Fys. Medd., 30:7 (1955), 1-23.

[9] W. Heisenberg, "Lie model in quantisation of nonlinear field equation", Nucl. Phys., 4 (1957), 532-563.

[10] Ф.А. Березин, "О модели Ли", Матем. сб., 60:4 (1963), 425-446; англ. пер.: F. A. Berezin, "On the Lee model", Amer. Math. Soc. Transl. Ser. 2, 56 (1966), 249-272.

[11] C. M. Bender, D. C. Brody, H. F. Jones, "Complex extension of quantum mechanics", Phys. Rev. Lett., 89:27 (2002), 270401.

[12] Л.С. Понтрягин, "Эрмитовы операторы в пространстве с индефинитной метрикой”, Изв. АН СССР. Сер. матем., 8:6 (1944), 243-280; англ. пер.: L.S. Pontryagin, "Hermitian operators in a space with an indefinite metric", Selected works. Vol. 1. Selected research papers, Classics of Soviet Mathematics, ed. R.V. Gamkrelidze, Gordon \& Breach Science Publishers, New York, 1986, 303-334.

[13] И. С. Иохвидов, М. Г. Крейн, "Спектральная теория операторов в пространствах с индефинитной метрикой”, Tp. MMO, 5 (1956), 367-432; англ. пер.: I. S. Iohvidov, M. G. Krein, "Spectral theory of operators in spaces with indefinite metric. I", Amer. Math. Soc. Transl. Ser. 2,13 (1960), 105-175; И. С. Иохвидов, М. Г. Крейн, "Спектральная теория операторов в пространствах с индефинитной метрикой. II", Tp. MMO, 8 (1959), 413-496; англ. пер.: I. S. Iohvidov, M. G. Krein, "Spectral theory of operators in spaces with indefinite metric. II", Amer. Math. Soc. Transl. Ser. 2, 34 (1963), 283-373.

[14] Ю.П. Гинзбург, И. С. Иохвидов, "Исследования по геометрии бесконечномерных пространств с билинейной метрикой”, УМH, 17:4 (1962), 3-56; англ. пер.: 
Yu. P. Ginzburg, I. S. Iokhvidov, "The geometry of infinite-dimensional spaces with a bilinear metric", Russian Math. Surveys, 17:4 (1962), 1-51.

[15] В.М. Бухштабер, Д. В. Лейкин, "Функциональные уравнения, определяющие умножение в непрерывном базисе Кричевера-Новикова", УМН, 61:1 (2006), 171-172; англ. пер.: V. M. Buchstaber, D. V. Leikin, "Functional equations defining multiplication in a continuous Krichever-Novikov basis", Russian Math. Surveys, 61:1 (2006), 165-167.

[16] В. М. Бухштабер, Д. В. Лейкин, "Законы сложения на якобианах плоских алгебраических кривых", Тр. МИАН, 251, Наука, М., 2005, 54-126; англ. пер.: V. M. Buchstaber, D. V. Leikin, "Addition laws on Jacobian varieties of plane algebraic curves", Proc. Steklov Inst. Math., 251 (2005), 49-120.

[17] И. М. Кричевер, "Спектральная теория "конечнозонных" нестационарных операторов Шредингера. Нестационарная модель Пайерлса", Функи. анализ и его прил., 20:3 (1986), 42-54; англ. пер.: I. M. Krichever, "Spectral theory of finite-zone nonstationary Schrödinger operators. A nonstationary Peierls model", Funct. Anal. Appl., 20:3 (1986), 203-214.

[18] Б. А. Дубровин, С. М. Натанзон, "Вещественные тэта-функциональные решения уравнения Кадомцева-Петвиашвили", Изв. АН СССР. Сер. матем., 52:2 (1988), 267-286; англ. пер.: В. A. Dubrovin, S. M. Natanzon, "Real theta-function solutions of the Kadomtsev-Petviashvili equation", Math. USSR-Izv., 32:2 (1989), 269-288.

[19] W. Koppelman, "Singular integral equations, boundary-value problems, and the Riemann-Roch theorem", J. Math. Mech., 10:2 (1961), 247-277.

[20] Ю. Л. Родин, "Краевая задача Римана для дифференциалов на римановых поверхностях", Ученые записки Пермского ун-та, 17:2 (1960), 83-85.

[21] P. G. Grinevich, A. Yu. Orlov, E. I. Schulman, "On the symmetries of the integrable systems", Important developments in soliton theory, Springer, Berlin, 1993, 283-301.

[22] В. А. Аркадьев, А. К. Погребков, М. К. Поливанов, "Сингулярные решения уравнения КдВ и метод обратной задачи", Записки науч. сем. ЛОМИ, 133 (1984), $17-37$.

[23] M. Adler, J. Moser, "On a class of polynomials connected with the Korteweg-de Vries equation", Comm. Math. Phys., 61:1 (1978), 1-30.

[24] M. M. Crum, "Associated Sturm-Liouville systems", Quart. J. Math. Oxford Ser. (2), 6:1 (1955), 121-127.

П. Г. Гриневич (P. G. Grinevich)

Институт теоретической физики

им. Л. Д. Ландау РАН

E-mail: pgg@landau.ac.ru

\section{С. П. Новиков (S. P. Novikov)}

Институт теоретической физики

им. Л. Д. Ландау РАН;

University of Maryland,

College Park

E-mail: novikov@ipst.umd.edu
Поступила в редакцию 24.06.2009 